\title{
Including Radiative Effects in an Entrainment Rate Formula for Buoyancy-Driven PBLs
}

\author{
Chin-Hoh Moeng, Peter P. Sullivan, and Bjorn Stevens \\ National Center for Atmospheric Research,* Boulder, Colorado
}

(Manuscript received 27 May 1997, in final form 15 June 1998)

\begin{abstract}
The effect of longwave radiative cooling at the planetary boundary layer (PBL) top in determining the entrainment rate was examined in this study. The entrainment rate equation that accounts for longwave radiative cooling can be formally derived from the following steps: 1) Derive the mean buoyancy budget in the thin layer between the averaged entrainment flux (i.e., the minimum buoyancy flux) level and the top of the entrainment zone where turbulent fluxes vanish. 2) Use the Deardorff entrainment closure assumption that the entrainment buoyancy flux is proportional to the vertically averaged buoyancy flux over the whole PBL, which is a generalized form of a widely accepted entrainment closure for the surface-heated convective PBL. This leads to an entrainment velocity that depends linearly on both the inverse of the interfacial Richardson number and the radiative flux divergence above the entrainment buoyancy flux level.

The relative importance of these two terms was examined through large eddy simulations (LESs) of several smoke-cloud-topped PBLs with various radiative forcings, radiative properties, temperature inversion strengths, and numerical advective schemes. These PBLs are driven only by cloud-top radiative cooling. The LESs were performed with a fine-grid nesting layer in the entrainment zone where the grid size is about $16 \mathrm{~m}$ and $8 \mathrm{~m}$ in the horizontal and vertical, respectively, which should be sufficient to resolve entrainment processes, at least for cases with weak capping inversions. The LESs showed that the contribution to entrainment rate from the radiative flux divergence term was either larger than or about equal to that of the interfacial Richardson number term. The LESs also showed that this radiative flux divergence occurs within cloudy regions below the local cloud tops.

The analysis was extended to the stratocumulus-topped PBL by using a stratocumulus-like smoke layer, and the result showed that the radiative flux contribution to the entrainment rate was still significant. Based on the physical understanding that this portion of the radiative flux divergence occurs within the upper half of cloud (or smoke) hummocks, the authors were able to analytically derive a relationship that links the radiative flux divergence to the cloud-top fluctuations, which were then empirically related to the interfacial Richardson number.
\end{abstract}

\section{Introduction}

Entrainment is perhaps the most challenging problem in planetary boundary layer (PBL) research. We do not understand very well what physical processes control entrainment and what physical variables determine the entrainment rate. The entrainment problem is particularly important for stratocumulus-topped PBLs. Through an entrainment instability mechanism (Lilly 1968; Deardorff 1980; Randall 1980a), stratocumulus may thin or dissipate totally. Entrainment may also strongly affect the drop size distribution near the cloud top, and hence cloud albedo and drizzle formation.

* NCAR is sponsored by the National Science Foundation.

Corresponding author address: Dr. Chin-Hoh Moeng, MMM Division, NCAR, P.O. Box 3000, Boulder, CO 80307-3000.

E-mail: moeng@ncar.ucar.edu
However, determining the entrainment rate is known to be one of the most difficult closure problems (e.g., Stage and Businger 1981; Randall 1984; Moeng 1987) in mixed-layer modeling of stratocumulus-topped PBL.

For the clear convective PBL with small wind shear, the entrainment rate normalized by the convective velocity scale is often assumed to be proportional to the inverse of an interfacial Richardson number, and the proportionality constant is often taken to be 0.2 . In section 2 we revisit this entrainment rate equation, which can be derived from the buoyancy jump condition along with the closure assumption that the entrainment buoyancy flux is proportional to the vertically averaged buoyancy flux over the whole PBL (which, in the limit of surface buoyancy driving only, is the same as assuming that the entrainment buoyancy flux is a fixed fraction of the surface buoyancy flux). From such a mathematical derivation, we are forced to define the interfacial Richardson number based on the buoyancy jump between the entrainment buoyancy flux level and the top of the entrainment zone where turbulent fluxes vanish, which 
is different from many previous investigators (e.g., Bretherton et al. 1999, hereafter BR99).

The stratocumulus-topped PBL turbulence can be driven by many forcings, such as surface heating, cloudtop longwave radiative and evaporative cooling, condensation, and wind shear. The most typical and longlasting stratocumulus-topped PBLs are dominated by cloud-top radiative cooling. We do not understand very well how entrainment processes vary between the surface-heated and the top-cooled PBLs. Questions as to how the entrainment rate depends on the cloud-top radiative forcing remain to be answered.

A heated debate took place in the late 1970s about the effect of distributed radiative cooling on entrainment (e.g., Deardorff 1976; Kahn and Businger 1979; Lilly and Schubert 1980; Randall 1980b; Stage and Businger 1981; Nieuwstadt and Businger 1984). In a mixed-layer framework, the cloud-top longwave radiation effect is inevitably split into two portions because of the averaging procedure. When the averaging is made over an Eulerian coordinate, ${ }^{1}$ one portion of the radiative flux divergence ends up in the conservation equation for the mixed-layer mean buoyancy, while the other portion enters into the entrainment rate equation. This led the above researchers to argue that these two portions of radiative flux divergence must play different roles in determining the entrainment rate. Because of their mathematical appearance, it looks as though the part in the conservation equation can directly generate turbulence and hence "indirectly" affect the entrainment rate, whereas the other portion "directly" affects the entrainment rate because it shows up in the entrainment rate equation. The physical interpretation these previous studies offered for the latter portion of the radiative flux divergence was that it cools the clear air above the cloud edge, and by making the clear air colder it reduces the local inversion strength and thus makes entrainment easier. We will show in this study that this physical interpretation is wrong.

We will show that physically both portions of the radiative flux divergence are located within cloudy air (although in nature there exists some clear-air longwave radiative cooling, but its magnitude is much smaller than the in-cloud longwave cooling we refer to in this study); physically, they play the same role in promoting turbulence and entrainment even though they show up in different mathematical equations after averaging. We will revisit an entrainment rate equation for a cloud-top

\footnotetext{
${ }^{1}$ Lilly and Schubert (1980) and Nieuwstadt and Businger (1984) argued that if an averaging is made over a vertical coordinate that follows the local cloud top, none of the in-cloud radiative flux divergence would show up in the entrainment rate equation. Here we prefer to use the concept of averaging over an Eulerian coordinate system because it is difficult, if not impossible, to observationally or numerically obtain the "entrainment" buoyancy flux averaged along the local cloud tops, and this flux may be quite different from the entrainment flux that is defined as in an Eulerian framework.
}

radiatively driven PBL that divides the entrainment rate, normalized by the convective velocity, into two terms: one inversely proportional to the interfacial Richardson number, and the other proportional to the radiative flux jump above the minimum buoyancy flux level, within the entrainment zone. We used large eddy simulation (LES) to check this entrainment rate equation and to examine the relative importance of these two terms. To provide a clear physical picture of this radiative flux divergence, we decide to use a smoke cloud to avoid the complications due to latent heating. A smoke cloud may exaggerate the importance of the radiative flux divergence, but it allows us to clearly demonstrate the physical origins of such flux divergence and so provides a basis for parameterizing this radiative flux effect.

Can the LES technique be used to predict entrainment rates accurately? An LES study by Moeng et al. (1996), which intercompared ten more or less independently developed LES computer codes for a stratocumulustopped PBL case, showed that the entrainment rate prediction is quite sensitive to the treatments of longwave radiation, numerics, and subgrid-scale (SGS) turbulence and condensation. That study showed that the range of the predicted entrainment rates among 10 different LES codes was as large as a factor of 8! BR99 carried out a further LES intercomparison study that eliminated most of the complications due to radiation and condensation treatments. In that study, a smoke-cloud case was chosen so that the uncertainty due to condensation and evaporation effects could be eliminated. And all LES codes used the same longwave radiation formulation to ensure that cloud-top cooling was comparable. That study gave a more promising result: if the vertical grid size is fine enough to resolve the horizontal variability of the local inversion height (i.e., cloud-top undulations or hummocks), all participating LESs give entrainment rates within $\pm 30 \%$ regardless of their treatments in the SGS turbulence (e.g., using different SGS length scale or different dissipation constant) and numerics (e.g., using monotone or nonmonotone schemes). We shall keep in mind this uncertainty of LES in our analysis.

Section 2 gives a review of the derivation of a commonly used entrainment rate equation for the clear convective PBL driven by surface heating only. In section 3 we extend the derivation to obtain an entrainment rate equation for the PBL that is driven by cloud-top radiative cooling, describe a suite of smoke-cloud LES solutions, use them to examine the entrainment rate equation, and provide a physical interpretation of the radiative flux divergence that appears in the entrainment rate equation. Section 4 extends our discussion to the marine stratocumulus-topped PBL. Section 5 gives a summary and draws conclusions.

\section{Review of the commonly used entrainment rate equation}

The virtual potential temperature is a conserved quantity in a dry adiabatic process, and thus the gov- 
erning equation of the mean virtual potential temperature $\Theta_{v}$ is

$$
\frac{\partial \Theta_{v}}{\partial t}=-\frac{\partial \overline{w \theta_{v}}}{\partial z}
$$

where $\overline{w \theta_{v}}$ is the virtual potential temperature flux (here we ignore the $g / T_{0}$ factor and refer to this term as the buoyancy flux) associated with turbulent motion. The capital-lettered $\Theta_{v}$ and the overbars represent ensemble averages. The quasi-steady state is defined as the state when the profile of the mean gradient of $\Theta_{v}$ remains unchanged in time; that is,

$$
\frac{\partial}{\partial t} \frac{\partial \Theta_{v}}{\partial z}=-\frac{\partial^{2} \overline{w \theta_{v}}}{\partial z^{2}}=0 .
$$

So under a quasi-steady state the buoyancy flux is linear with height.

Integrating (2.1) over a finite-depth, thin layer at the PBL top, from the entrainment buoyancy flux level $\left[z_{\mathrm{fl}}\right.$, following the notation used by Lock and MacVean (1999, hereafter LM99) to stress the entrainment flux level] to the top of the entrainment zone $\left(z_{\mathrm{fl}}^{+}\right)$where turbulence fluxes vanish and making the "thin layer assumptions" to be described later, we obtain the buoyancy jump condition

$$
\left.\overline{w \theta_{v}}\right|_{z_{\mathrm{fl}}}=-w_{e} \Delta \Theta_{v},
$$

where $w_{e} \equiv d z_{\mathrm{fl}} / d t-W_{\mathrm{LS}}$ is the entrainment velocity and $W_{\mathrm{LS}}$ is the environmental large-scale vertical motion. This jump condition describes the mean buoyancy budget within the entrainment zone: cooling due to the turbulent flux jump balances warming due to entrainment. The minimum buoyancy flux at the cloud top is associated with entrainment, and thus is also referred to as the entrainment buoyancy flux. Note that the jump condition $\Delta$ defined in this paper is strictly based on the above mathematical derivation; it represents the difference of quantities between the entrainment flux level $z_{\mathrm{fl}}$ and a level above where the turbulence vanishes. Such defined $\Delta \Theta_{v}$, therefore, does not necessarily represent the full inversion strength, which is traditionally defined as the total buoyancy jump between the well-mixed layer and that above the entrainment zone.

The "thin layer assumptions" used to derive (2.3) are (a) both $z_{\mathrm{fl}}$ and $z_{\mathrm{fl}}^{+}$levels are lifted up at the same rate (i.e., $d z_{\mathrm{fl}} / d t=d z_{\mathrm{fl}}^{+} / d t$ ) and (b) the time-rate-of-change of $\int_{z_{\mathrm{fl}}}^{z_{1}^{+}} \Theta_{v} d z$ is negligible (e.g., Lilly 1968). Recently, vanZanten et al. (1999) and Sullivan et al. (1998, hereafter SU98) showed that the second assumption does not hold for their surface-heating-driven PBL simulations, which is understandable because both surface heating and entrainment warming tend to increase the magnitude of $\int_{z f l}^{z_{f 1}^{+}} \Theta_{v} d z$. This, however, may not be the case for the radiative-cooling-driven PBL because the effects of radiative cooling and entrainment warming tend to cancel each other. We will test the above assumptions for our smoke-cloud simulations in section 3 .
To obtain an entrainment rate equation from (2.3), a closure assumption is needed. A closure assumption, proposed by Deardorff (1976), is to set the entrainment buoyancy flux proportional to the layer-averaged buoyancy flux; that is,

$$
\left.\overline{w \theta_{v}}\right|_{z_{\mathrm{fl}}}=-A^{\prime} \int_{0}^{z_{\mathrm{fl}}} \overline{w \theta_{v}} d z / z_{\mathrm{fl}}
$$

where $A^{\prime}=0.5$ is assumed by Deardorff (1976). This closure is physically plausible because entrainment fluxes depend mainly on the turbulence intensity, and turbulence intensity is measured mainly by the whole layeraveraged buoyancy flux, at least for the buoyancy-driven PBL.

In the limit of the clear convective PBL where the surface buoyancy $\left(\overline{w \theta_{v}}\right)_{0}$ is the only source of turbulence, the buoyancy flux is linear with height as shown in $(2.2)$, and thus $\int_{0}^{z \mathrm{ff}} \overline{w \theta_{v}} d z / z_{\mathrm{fl}}=\left[\left.\overline{w \theta_{v}}\right|_{z_{\mathrm{fl}}}+\left(\overline{w \theta_{v}}\right)_{0}\right] / 2$. So (2.4) reduces to

$$
\left.\overline{w \theta_{v}}\right|_{z_{\mathrm{f} 1}}=-\left(\frac{A^{\prime}}{2+A^{\prime}}\right)\left(\overline{w \theta_{v}}\right)_{0}=-A\left(\overline{w \theta_{v}}\right)_{0} .
$$

This is a commonly used entrainment rate equation for the clear-convective PBL (e.g., Carson 1973). It states that the entrainment buoyancy flux is proportional to the surface buoyancy flux. The proportionality constant $A\left[\equiv A^{\prime} /\left(2+A^{\prime}\right)\right]$ is often set to 0.2 . The observed values of $A$ may vary between 0.1 and 0.5 , but the larger observed $A$ values are likely due to large shear effects, as demonstrated in Moeng and Sullivan (1994). For the free convective PBL, the value of 0.2 is reasonable (Stull 1976) although Sorbjan (1996) suggested that $A$ depends on the lapse rate above the capping inversion. Recently, based on their clear convective PBL simulations, SU98 also showed that the $A$ value (which in their notation is $A_{w \theta}$ ) may increase with the interfacial Richardson number $\mathrm{Ri}$ for $\mathrm{Ri}<30$ but then reaches an asymptote of about 0.17 for large $\mathrm{Ri}$.

Using (2.5) in (2.3) yields the well-known entrainment rate equation for the clear convective PBL (when shear is negligibly small):

$$
\frac{w_{e}}{w_{*}}=\frac{A}{\mathrm{Ri}},
$$

where

$$
w_{*} \equiv\left(\frac{g}{T_{0}}\left(\overline{w \theta_{v}}\right)_{0} z_{\mathrm{fl}}\right)^{1 / 3}
$$

is the convective velocity defined by Deardorff (1974), and the interfacial Richardson number of the inversion

$$
\mathrm{Ri} \equiv\left(g / T_{0}\right) z_{\mathrm{fl}} \Delta \Theta_{v} / w_{*}^{2}
$$

(e.g., Deardorff 1981). From the above derivation, we are forced to define $\mathrm{Ri}$ based on $\Delta \Theta_{v}$ - the buoyancy 
jump between the entrainment buoyancy flux level $z_{\mathrm{fl}}$ and the top of the entrainment zone $z_{\mathrm{fl}}^{+}$.

Equation (2.4) can be seen as a generalized closure assumption of (2.5), which may then be applied to any buoyancy-driven PBL, including not only the surfaceheating driven PBL, but also the cloud-top radiativecooling-driven PBL or the combination of the two. In the next section, we will use exactly the same procedure to derive an entrainment rate equation for any buoyantly driven PBLs, but will use the radiatively driven PBL as an example.

\section{Entrainment velocity in radiatively driven smoke-cloud-topped PBLs}

\section{a. Derivation of the entrainment rate equation}

In the presence of radiation, buoyancy is not conserved and thus the buoyancy flux is no longer linear with height even in a quasi-steady state. It is the net heat flux $\overline{w \theta_{v}}+\overline{F_{R}} / \rho_{0} c_{p}$ that is linear with height because the governing equation for the mean buoyancy is now

$$
\frac{\partial \Theta_{v}}{\partial t}=-\frac{\partial \overline{w \theta_{v}}}{\partial z}-\frac{1}{\rho_{0} c_{p}} \frac{\partial \overline{F_{R}}}{\partial z},
$$

where $\overline{F_{R}}$ is the horizontally averaged longwave radiative flux, $\rho_{0}$ is the reference air density, and $c_{p}$ is the specific heat of dry air at constant pressure.

We will now follow the same procedure as in section 2 to derive an entrainment rate equation for the radiatively driven PBL. Again, in order to obtain a jump condition that relates the entrainment buoyancy flux to the entrainment velocity, we integrate (3.1) from the most negative buoyancy flux (i.e., entrainment buoyancy flux) level, $z_{\mathrm{ff}}$, to the level above the PBL top where the buoyancy flux vanishes, $z_{\mathrm{fl}}^{+}$, and use the "thin layer assumptions." These manipulations lead to the heat budget within the thin jump layer:

$$
\left.\overline{w \theta_{v}}\right|_{z_{\mathrm{fl}}}-\frac{1}{\rho_{0} c_{p}} \Delta F_{R}=-w_{e} \Delta \Theta_{v},
$$

where $\Delta F_{R} \equiv \overline{F_{R}\left(z_{\mathrm{fl}}^{+}\right)}-\overline{F_{R}\left(z_{\mathrm{fl}}\right)}$ is the jump of longwave radiation flux between the $z_{\mathrm{fl}}$ and $z_{\mathrm{fl}}^{+}$levels. Equation (3.2) describes the heat budget in this thin jump layer: cooling due to turbulent flux divergence and radiative flux divergence balances warming due to entrainment. Note again that $\Delta \Theta_{v}$ here represents the $\Theta_{v}$ difference between $z_{\mathrm{fl}}$ and $z_{\mathrm{fl}}^{+}$, and so does not necessarily represent the whole inversion strength, which is often defined as the total buoyancy jump above the well-mixed layer.

We now apply the same closure assumption as that for the bottom-heating driven convective PBL (2.4), which we repeat here for clarity:

$$
\left.\overline{w \theta_{v}}\right|_{z \mathrm{fl}}=-A^{\prime} \int_{0}^{z_{\mathrm{fl}}} \overline{w \theta_{v}} d z / z_{\mathrm{fl}} .
$$

Using (3.3) in (3.2) yields

$$
\frac{w_{e}}{w_{* c}}=\frac{A}{R_{i c}}+\frac{\Delta F_{R}}{\rho_{0} c_{p} w_{* c} \Delta \Theta_{v}},
$$

where $A \equiv A^{\prime} /\left(2+A^{\prime}\right)$ and $w_{* c}$ is the generalized convective velocity defined by Deardorff (1976),

$$
w_{* c} \equiv\left[\left(2+A^{\prime}\right)\left(g / T_{0}\right) \int_{0}^{z_{\mathrm{fl}}} \overline{w \theta_{v}} d z\right]^{1 / 3},
$$

which equals (2.7) for bottom-heated convective PBL cases and the convective Richardson number is

$$
R_{i c} \equiv\left(g / T_{0}\right) z_{\mathrm{fl}} \Delta \Theta_{v} / w_{* c}^{2} .
$$

The closure (3.3) is similar to that proposed by Schubert (1976) if the minimum buoyancy flux occurs at the cloud top.

The entrainment rate equation (3.4) reduces to (2.6) when there is no radiative forcing. The extra term in (3.4) is proportional to the longwave radiative flux jump between the entrainment buoyancy flux level and above it where the turbulence vanishes. So, we see there is indeed some portion of radiative flux divergence that occurs within the entrainment zone. The questions are, how important is this divergence in determining the entrainment rate and what is the physical interpretation of this radiative flux divergence term? To answer these questions we use large-eddy simulations.

\section{b. The smoke-cloud LESs}

To simplify our discussion, we will focus on radiatively driven smoke-cloud PBLs. Possible applications to the stratocumulus-topped PBL will be discussed in section 4 . By smoke cloud, we mean a layer that radiates as liquid water but does not go through a phase change. The use of smoke cloud as a prototype stratocumulus was suggested by Lilly (1968) and has been used by many investigators (e.g., Schubert et al. 1979, Nieuwstadt and Businger 1984; Moeng et al. 1992; BR99). Like stratocumulus, a smoke cloud also emits longwave radiation as a graybody. This results in a sharp divergence of the longwave radiation flux near the cloud top, leading to strong radiative cooling that can buoyantly drive turbulence, just as in the stratocumulus-topped PBL. Thus, a smoke-topped PBL-shares with the stratocumulus-topped PBL the essential features of turbulence and entrainment driven by radiative cooling.

The smoke-cloud case considered here has no surface heating, no wind shear, and free slip (i.e., no stress) bottom and top boundary conditions. This case was designed for and used in the second intercomparison study of the GEWEX Cloud System Study (GCSS) Boundary Layer Cloud Working Group, which focused on entrainment rate prediction (BR99). In the smoke cloud, the longwave radiative cooling at the cloud top is the only source of turbulence.

A total of nine smoke-cloud cases were simulated (as summarized in Table 1). (The CONTR* run listed in 
TABLE 1. LES parameters.

\begin{tabular}{lcccl}
\hline \hline Experiment & $\begin{array}{c}F_{0}(\mathrm{~W} \\
\left.\mathrm{m}^{-2}\right)\end{array}$ & $\begin{array}{c}\text { Initial } \\
\text { inversion } \\
\text { strength }(\mathrm{K})\end{array}$ & $\begin{array}{c}\kappa_{R}\left(\mathrm{~m}^{2}\right. \\
\left.\mathrm{kg}^{-1}\right)\end{array}$ & $\begin{array}{c}\text { Vertical } \\
\text { derivative } \\
\text { scheme }\end{array}$ \\
\hline CONTR & 60 & 1.25 & 0.02 & Centered finite \\
FR30 & 30 & 1.25 & 0.02 & Centered finite \\
XKR-S & 60 & 1.25 & 0.01 & Centered finite \\
XKR-L & 60 & 1.25 & 0.04 & Centered finite \\
INV2 & 60 & 2.50 & 0.02 & Centered finite \\
INV3 & 60 & 7.00 & 0.02 & Centered finite \\
INV3-MFC & 60 & 7.00 & 0.02 & Monotone \\
MFC & 60 & 1.25 & 0.02 & Monotone \\
CONTR* & 60 & 1.25 & 0.02 & Centered finite \\
\hline
\end{tabular}

the table will be described in section 4.) Variations among CONTR, FR30, XKR_L, XKR_S, INV2, and INV3 resulted from different prescriptions of the radiative forcing, the depth of the radiative cooling zone, or the temperature inversion strength, as listed in Table 1. The MFC and INV3-MFC cases are designed to check the sensitivity to numerical schemes. Representations of vertical derivatives by second-order centered finite-differencing schemes is known to lead to overshoots in smoke concentration (and other scalar fields) near the inversion where strong vertical gradients exist. Thus, to test the solution's sensitivity to this advection scheme we ran MFC and INV3-MFC using a second-order mass flux-corrected upwind scheme with monotone properties (Beets and Koren 1996; Koren 1993) for the calculation of the vertical derivatives of the smoke and the $\theta_{v}$ fields. This advection scheme prevents spurious oscillations in the smoke field near the inversion by adding an implicit numerical viscosity.

Our sounding is the same as that of the GCSS smokecloud case (BR99), except that for most cases we choose a much smaller temperature inversion. Most cases have an inversion that is 3 to 6 times smaller than the GCSS case, except for the two INV3 cases, which have the same inversion strength as the GCSS study. The purpose of choosing weaker capping inversions (which produce larger cloud-top undulations) is to have better resolved entrainment processes and a more accurate entrainment rate prediction. We also adopt the nested-grid LES code developed by Sullivan et al. (1996), in which an outer (coarse) grid covers a $3200 \mathrm{~m} \times 3200 \mathrm{~m} \times 1250 \mathrm{~m}$ domain with $64 \times 64 \times 50$ grid points, with a nested (finer) grid covering a $3200 \mathrm{~m} \times 3200 \mathrm{~m} \times 250 \mathrm{~m}$ domain at the entrainment zone with $192 \times 192 \times 30$ grid points. Thus our grid mesh in the entrainment region is three times finer in the horizontal directions and also three times finer in the vertical compared to the standard resolution run of the GCSS study. The time step is about $3 \mathrm{~s}$ with the explicit third-order accurate Runge-Kutta scheme also described in Sullivan et al. (1996).

As in the GCSS study, we used a simple exponential formulation to compute the longwave radiation flux:
$F_{R}(x, y, z)=F_{0} \exp \left[-\rho_{0} \kappa_{R} \int_{z}^{\infty} s\left(x, y, z^{\prime}\right) d z^{\prime}\right]$,

where $\int_{z}^{\infty} s\left(x, y, z^{\prime}\right) d z^{\prime}$ is the vertically integrated smokecloud concentration above the reference level $z ; \kappa_{R}$, which controls the depth of the longwave radiative cooling zone, is taken to be constant following BR99; and $F_{0}$ is the net longwave radiation flux at the top of the numerical domain representing the maximum rate at which energy can be extracted. We vary the magnitudes of $\kappa_{R}$ and $F_{0}$ among our LES cases as described in Table 1. Use of (3.7) implies that all clear-air radiation is excluded. We set $\rho_{0}=1.1436 \mathrm{~kg} \mathrm{~m}^{-3}$ in the radiation calculation to be consistent with the GCSS intercomparison study (BR99), even though the reference air density in our LES governing equation (which is a Boussinesq model; see Moeng 1984) is hardwired to $1 \mathrm{~kg}$ $\mathrm{m}^{-3}$. Thus, our conversion of buoyancy flux units from $\mathrm{W} \mathrm{m} \mathrm{m}^{-2}$ to $\mathrm{m} \mathrm{K} \mathrm{s}^{-1}$ is simply a multiplication factor of 1000 , for $c_{p}=1000 \mathrm{~J} \mathrm{~K}^{-1} \mathrm{~kg}^{-1}$.

We ran each case for 150 simulated minutes, which is about 11 large-eddy-turnover times. The three-dimensional instantaneous flow fields were stored at 5-min intervals over the last $80 \mathrm{~min}$ of the simulation, so that 17 LES flow fields were available for analysis in this study. We found that averaging over the 17 flow fields is adequate for the LESs studied here.

\section{c. LES results}

Figure 1 shows the temperature contours at the inversion and the flow velocity vectors from the CONTR run. In the upper-right corner, we also plot the grid mesh in the entrainment zone. Compared to the grid mesh, the cloud-top fluctuations are clearly well resolved as evidenced from the several entrainment events at $y \sim$ $(400,650,1000$, and 2600) $\mathrm{m}$ where wisps of warm air are being incorporated into the PBL.

In Fig. 2, we plot a sequence of snapshots of the event at $y=2600 \mathrm{~m}$ at $\sim 30 \mathrm{~s}$ time interval. This figure illustrates how warm air is incorporated into the PBL at the edge of a cloud-top hummock associated with a strong updraft. Based on many LESs of buoyantly driven PBLs, we see that strong updrafts are the main driver of the entrainment process, as discussed in more detail by SU99.

\section{1) THE MEAN SMOKE-CLOUD-TOP HEIGHT}

We need to find the entrainment buoyancy flux level, $z_{\mathrm{f}}$, in order to estimate the jump conditions and the entrainment rate. The most straightforward way to determine $z_{\mathrm{ff}}$ is to identify the level of the most negative buoyancy flux in each (simulated) instantaneous, horizontally averaged buoyancy flux profile. However, $z_{\mathrm{fl}}$ defined in this manner fluctuates greatly in time, as shown in Fig. 3 (these fluctuations are much more pro- 


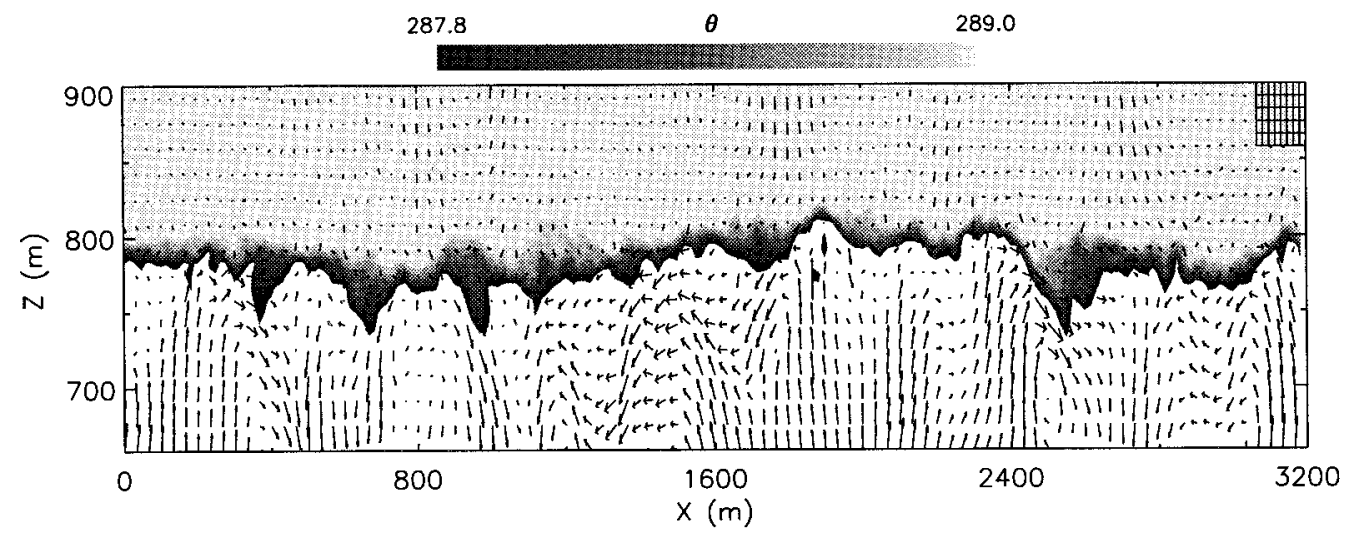

FIG. 1. A vertical cross section showing the temperature field near the boundary layer top (temperatures below $287.8 \mathrm{~K}$ are shown as white in this color table) and flow velocity vectors from the CONTR run. Several wisps observed in this plot may be responsible for most of the entrainment.

nounced for the clear convective PBL, as shown in SU98 and LM99, and are likely due to the sampling problem of the limited horizontal domain size), and hence is difficult to use for our analysis. (At certain instantaneous times, the negative buoyancy flux at the cloud top may disappear all together, e.g., at $\sim 105$ min of the XKR_S simulation. This makes the use of such defined $z_{\mathrm{fl}}$ more difficult.)

Here we used an alternative method. We calculated the mean-smoke-cloud-top height based on the smoke concentration field and used it to represent $z_{\mathrm{ff}}$. Following BR99, we first searched for the local cloud-top heights $z_{\text {top }}(x, y)$ as the levels where the smoke concentration equals 0.5 at each grid column. (The initial condition of the smoke concentration is 1 inside the PBL and 0 above. Thus, 0.5 is the mean of the initial maximum and minimum concentration values). We then horizontally averaged these local cloud-top heights to find the mean-smoke-cloud-top height, $z_{i}$, at each recorded time record. The time evolution of $z_{i}$ calculated in this manner is given in Fig. 4. Compared to Fig. 3, we found $z_{\mathrm{fl}}$ fluctuates about the $z_{i}$ level for most of the simulations, but for those with stronger capping inversions (e.g., INV2, INV3, and INV3-MFC), $z_{\mathrm{fl}}$ is more likely to stay below $z_{i}$ throughout the simulation time; the latter feature was also found in LM99.

For most LES runs, $z_{i}$ is nearly linear in time and hence computing $w_{e}\left(\equiv d z_{i} / d t\right)$ is straightforward. For some, like the MFC run, however, the slope changed over the last 80 min of simulation time. We estimated its entrainment rate averaged over the whole analysis time period and also over the last 40-min time period, and found the difference to be about $16 \%$. We consider this difference to be within the uncertainty of LES.

We found from the time-averaged buoyancy flux profiles, later shown in Fig. 8, that $z_{i}$, after time averaging, coincides with the time-averaged $z_{\mathrm{fl}}$ except for the cases with stronger capping inversions. Thus, we will first use $z_{i}$ to approximate $z_{\mathrm{fl}}$ for our analysis but will perform a sensitivity study on this approximation later.

The time evolution of the layer-averaged buoyancy flux $B \equiv \int_{0}^{z \mathrm{fl}} \overline{w \theta_{v}} d z / z_{\mathrm{fl}}$ is given in Fig. 5 for all cases; it reaches an asymptote during the last $\sim 60 \mathrm{~min}$ of the analysis time period.

\section{2) Statistical PROFiles}

Figure 6 shows the following statistics of the CONTR case: (a) mean virtual potential temperature $\Theta_{v}$, (b) turbulent kinetic energy (TKE), (c) buoyancy flux $\overline{w \theta_{v}}$, (d) longwave radiative flux $\overline{F_{R}}$, (e) total heat flux $\overline{w \theta_{v}}+$ $\overline{F_{R}} /\left(\rho_{0} c_{p}\right)$, and (f) smoke flux. These averaged statistics were constructed in a three-step procedure: (a) individual time records of turbulence quantities were averaged over $x-y$ planes, (b) horizontally averaged statistics were interpolated onto a vertical grid normalized by the mean-cloud-top height $z_{i}$ at the time of the record, and then (c) the height-normalized statistics profiles were averaged over the 17 time records.

As we can see from Figs. 6b, 6c, and 6f, most of the TKE and turbulent fluxes reside in the resolved scales of the simulation; only a small portion comes from the subgrid scales. Figure 6e shows that the total heat flux is nearly linear with height within the well-mixed layer, again indicating that the simulated turbulent field has reached a quasi-steady state. From the normalized buoyancy flux profile, we see that the level of the most negative flux is at the $z_{i}$ level in this case, so $z_{\mathrm{fl}}=z_{i}$ is a good approximation. Figure of shows the total smoke flux; it is not exactly linear with height, but close enough for our analysis.

In order to see the cloud-top jump more clearly, we present in Figs. 7 and 8 the vertical profiles of $\Theta_{v}, \overline{w \theta_{v}}$, and $\overline{F_{R}}$ from $z=0.9 z_{i}$ to $z=1.1 z_{i}$. We can see clearly that some portion, albeit small, of the horizontally averaged radiative flux divergence occurs above the $z_{i}$ (or 


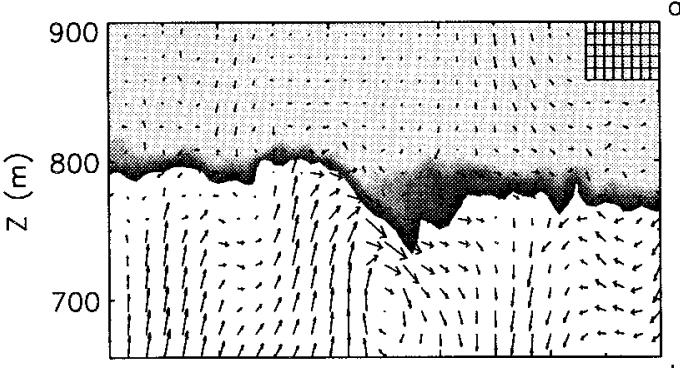

a)

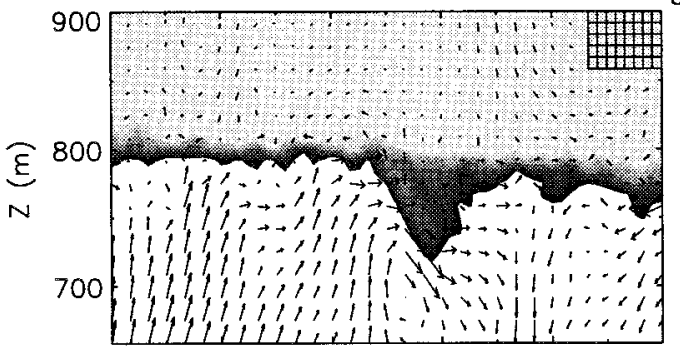

b)

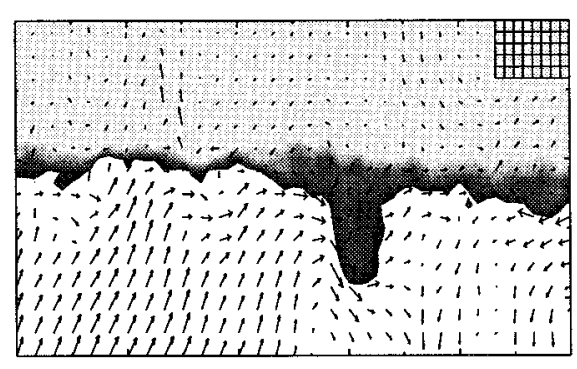

e)

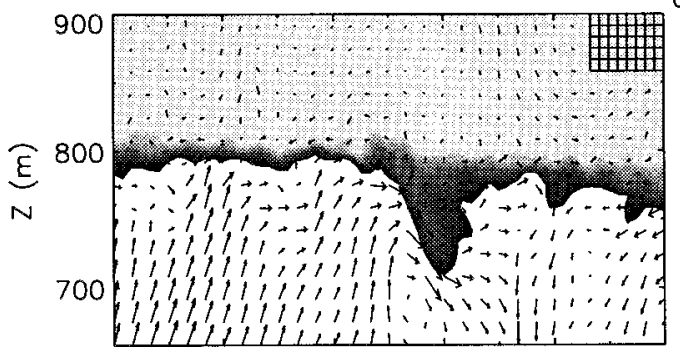

c)
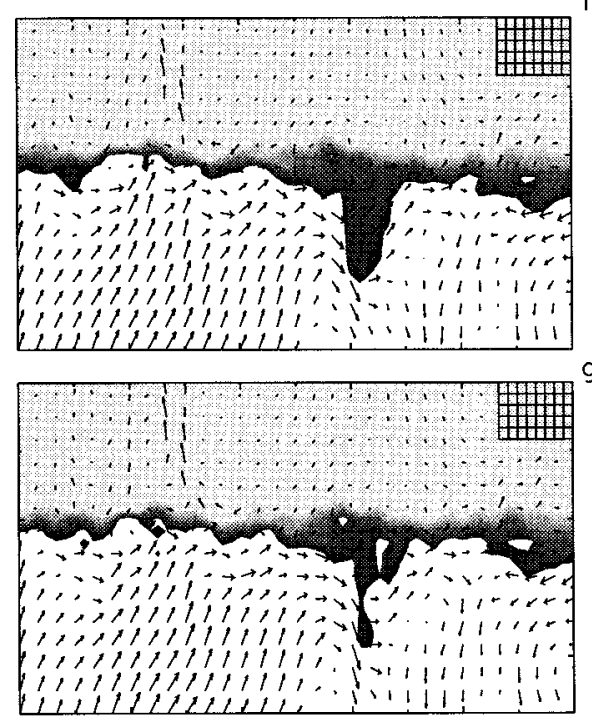

g)
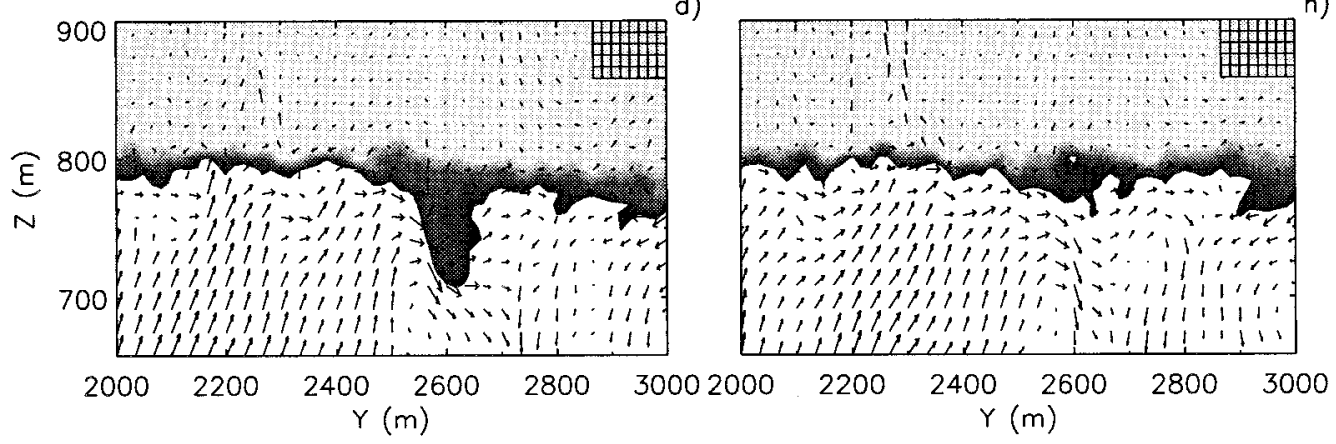

FIG. 2. Same as Fig. 1 but for a limited domain, from $y=2000-3000$ m. (a)-(h) Time sequence of snapshots.

$z_{\mathrm{f}}$ ) level. It exists within the entrainment zone. (Simulations with centered finite differencing reveal the two known spurious features: above the level where the turbulent flux is supposed to vanish, there exists a small positive buoyancy flux and a radiative flux that does not converge to $F_{0}$. These make centered finite differencing less appealing than monotone schemes, but we would like to point out here that monotone schemes also have spurious numerical flaws, although they are not as readily apparent.)

To calculate the jump conditions $\Delta \Theta_{v}$ and $\Delta F_{R}$, we first set $z_{\mathrm{fl}}=z_{i}$ and $z_{\mathrm{fl}}^{+}=1.05 z_{i}$ for all cases, where $1.05 z_{i}$ is the location where the virtual potential temperatures remain unchanged throughout the simulations for most cases. (Because the jump conditions and $R_{i c}$ depend on the definition of $z_{\mathrm{fl}}$ and $z_{\mathrm{fl}}^{+}$we will perform a sensitivity study that uses different heights to check if our conclusion still holds.) We then estimate the timeaveraged mean smoke-cloud-top height (from Fig. 4); the entrainment rate (i.e., the averaged slope of the curves in Fig. 4); the layer-averaged buoyancy flux over the whole PBL; $B$ (from Fig. 5); the entrainment buoyancy flux (from Fig. 8); and the jumps $\Delta \Theta_{v}$ (from Fig. 7) and $\Delta F_{R}$ (from Fig. 8). These values are given in Table 2. Inserting these values into (3.5) and (3.6), we can compute $w_{* c}$ and $\mathrm{Ri}_{c}$, which are given in Table 3 .

Table 2 shows that the radiative flux jump above $z_{\mathrm{fl}}$, $\Delta F_{R}$, is only a small fraction of the total radiative flux 


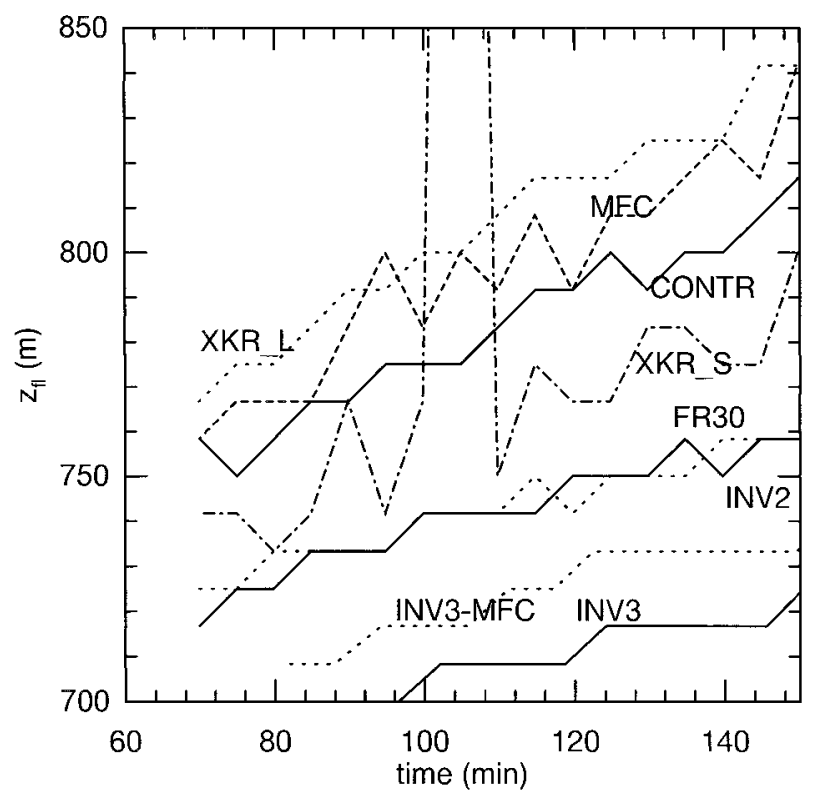

FIG. 3. Time evolution of $z_{\mathrm{fl}}$ from the eight smoke-cloud LESs.

jump across the cloud top; it is less than $20 \%$ of $F_{0}$ for most of the cases. This fraction becomes larger with thinner radiative cooling zone (comparing XKR_L to XKR_S), weaker inversion strength (comparing CONTR to INV2 and INV3), and the use of a monotone advection scheme (comparing MFC to CONTR).

\section{3) Examining equations (3.2), (3.3), And (3.4)}

The jump condition (3.2) was derived based on the "thin layer assumptions." In our LESs the depth of the actual jump layer ranges, as seen from Fig. 8, from $0.01 z_{i}$ (such as FR30) to about $0.06 z_{i}$ (such as MFC). Using the LES solutions we calculated both right- and left-hand sides of (3.2), which are plotted in Fig. 9. All LES data points lie close to the diagonal, indicating that the jump condition (3.2) is quite satisfied for all cases despite the use of the "thin layer assumptions." This suggests that the jump-layer temperature, $\int_{z \text { ff }}^{+} \Theta_{v} d z$, in these radiative-cooling-driven smoke-topped PBLs is nearly constant in time, unlike the surface-heated PBL where the jump-layer temperature increases significantly in time as demonstrated by SU98. We speculate that cloud-top radiative cooling nearly cancels out entrainment warming within this thin jump layer.

Next we check the closure assumption (3.3) in Fig. 10 , which plots the ratio $A^{\prime}$ of the entrainment buoyancy flux to the layer-averaged buoyancy flux, as a function of the layer-averaged buoyancy flux. [The error bars in Fig. 10 represent the standard deviations (in time) of $A^{\prime}$; these time variations result from the fact that the predicted entrainment buoyancy flux fluctuates greatly in time. For some LESs, the entrainment buoyancy flux may even become slightly positive for some short pe-

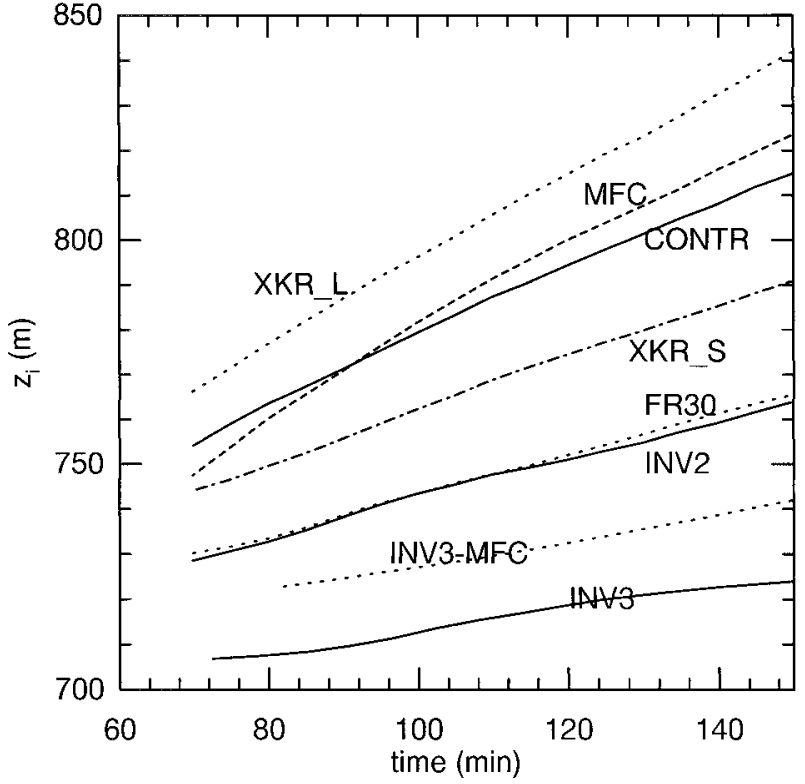

FIG. 4. Time evolution of $z_{i}$ from the eight smoke-cloud LESs.

riods of time. Because the frequency of these fluctuations is approximately $w_{e} / \Delta z$, where $\Delta z$ is the vertical grid size, we suspect that the vertical grid discretization is the main cause for the large time fluctuations in the predicted buoyancy flux.] Overall, the $A^{\prime}$ value in (3.3) is close to 0.5 except in the INV3-MFC run.

Both INV3-MFC and INV3 have the strongest capping inversion (7 K jump in the initial temperature field) among all cases studied here, and hence their cloud-top undulations are not as well resolved as those with weak-

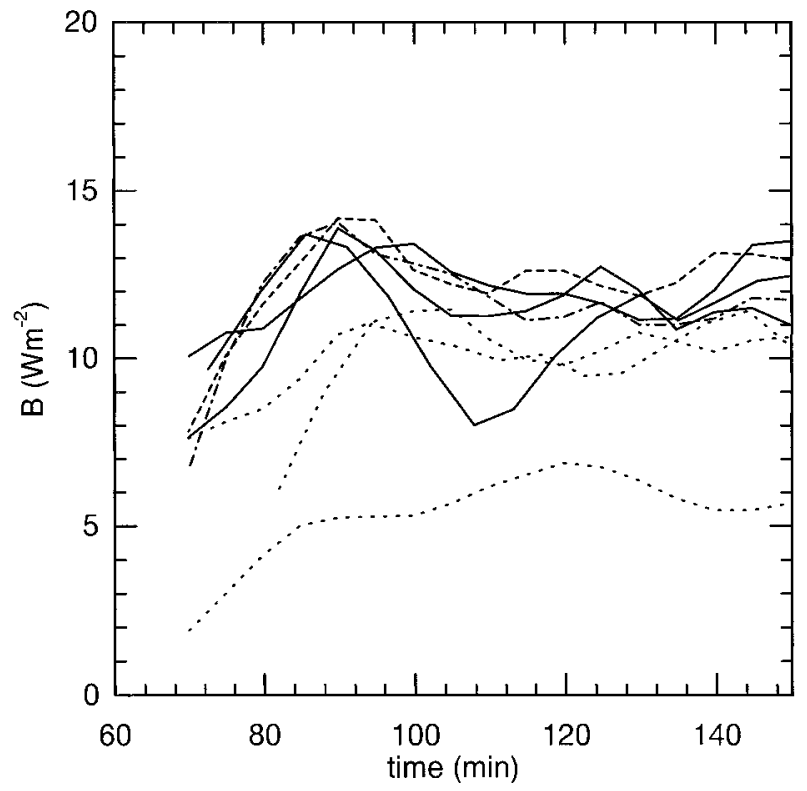

FIG. 5. Time evolution of the layer-averaged buoyancy flux from the eight smoke-cloud LESs. 

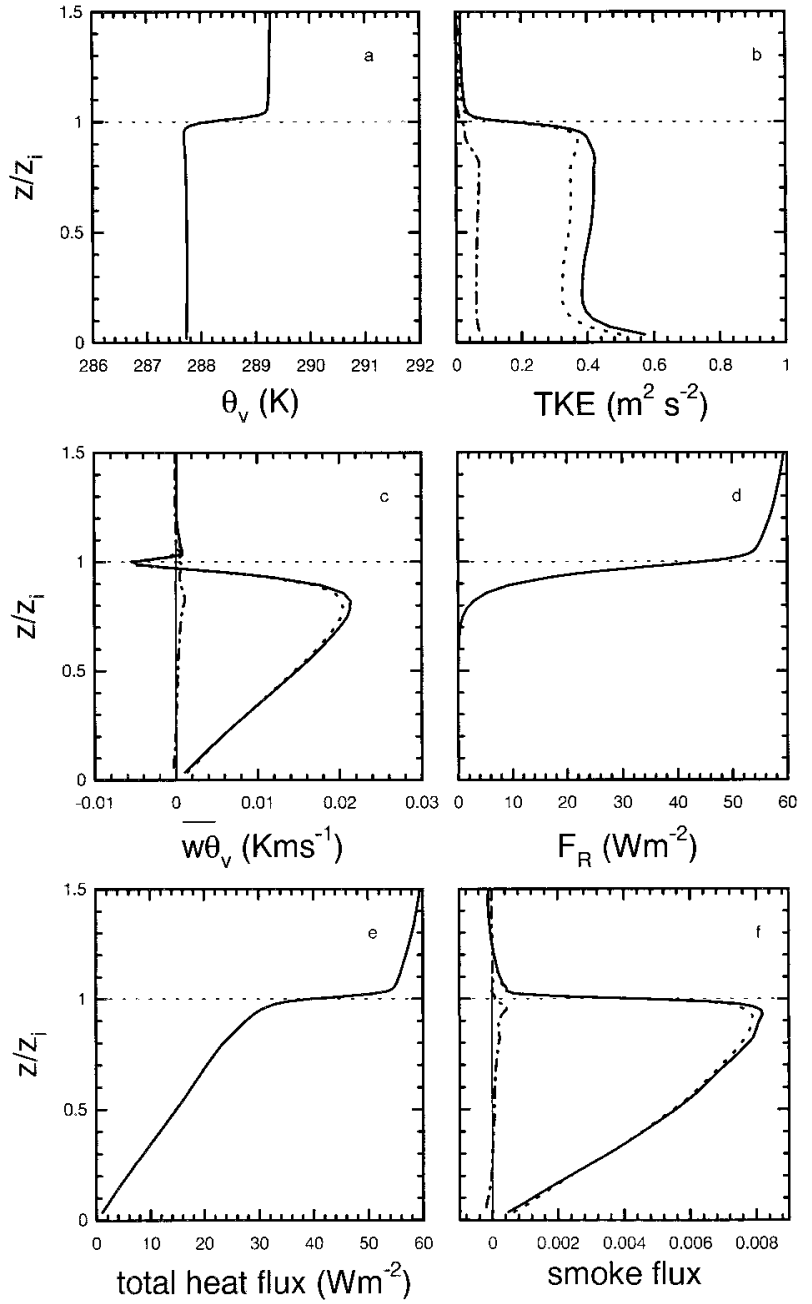

FIG. 6. Vertical distributions of (a) the mean virtual potential temperature; (b) the resolved (dotted), subgrid-scale (dashed-dotted), and total (solid) turbulence kinetic energy; (c) the resolved (dotted), subgrid-scale (dashed-dotted), and total (solid) buoyancy fluxes; (d) the longwave radiative flux; (e) sum of the total buoyancy and longwave radiative fluxes; and (f) the resolved (dotted), subgrid-scale (dasheddotted), and total (solid) smoke flux, from the CONTR run.

er capping inversion (e.g., that shown in Figs. 1 and 2). This makes their results on the entrainment-related quantities less reliable. One important point to note is that the only difference between these two LESs is the use of the advection scheme for the scalar fields-INV3 uses centered finite differencing and INV3-MFC a monotone scheme. Comparing these two runs shows that a monotone advection scheme tends to produce a larger numerical diffusion (as widely recognized and also evidenced by the more smoke produced in the entrainment zone), and hence a larger entrainment buoyancy flux. This numerical effect is more pronounced with a stronger capping inversion. We are currently examining the numerical effects on the entrainment-buoyancy-flux prediction from LES.

Applying $A^{\prime}=0.5$ for all cases, we plot in Fig. 11
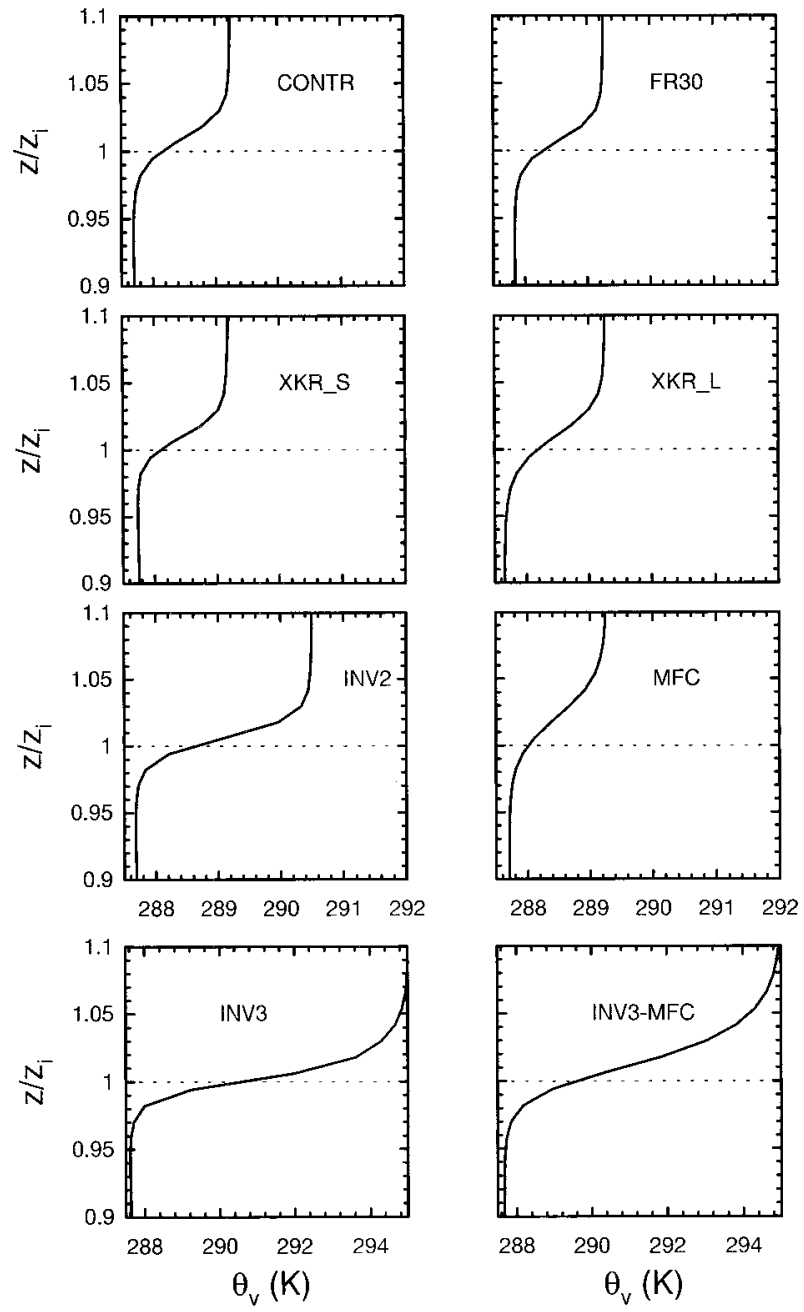

FIG. 7. The mean virtual potential temperature in the layer between $0.9 z_{i}$ and $1.1 z_{i}$ for all eight smoke-cloud cases. The dotted lines indicate the bottom of the jump layer.

the predicted entrainment rate normalized by $w_{*_{c}}$ (which is given in the third column of Table 3 ) against the sum of the two right-hand-side terms in (3.4). All data points lie very close to the diagonal line, showing that the entrainment rate equation works quite well for all eight simulated smoke-cloud cases. The two terms on the right-hand side are shown in the fourth and fifth columns of Table 3, respectively. We see that the $\Delta F_{R}$ term is larger than the $A / \mathrm{Ri}_{c}$ term for most of these smokecloud cases, despite the fact that $\Delta F_{R}$ is only a small fraction of $F_{0}$ for most of the cases. The relative contribution of the radiation term to the entrainment rate, compared to the $A / \mathrm{Ri}_{c}$ term, is larger for thinner radiative cooling zone, weaker inversion, and the use of a monotone advection scheme.

Despite the numerical effect, there is one thing we can learn from the strong capping inversion cases. Even with the much larger $A^{\prime}$ value in the INV3-MFC case, the entrainment rate equation (3.4) still holds quite well 

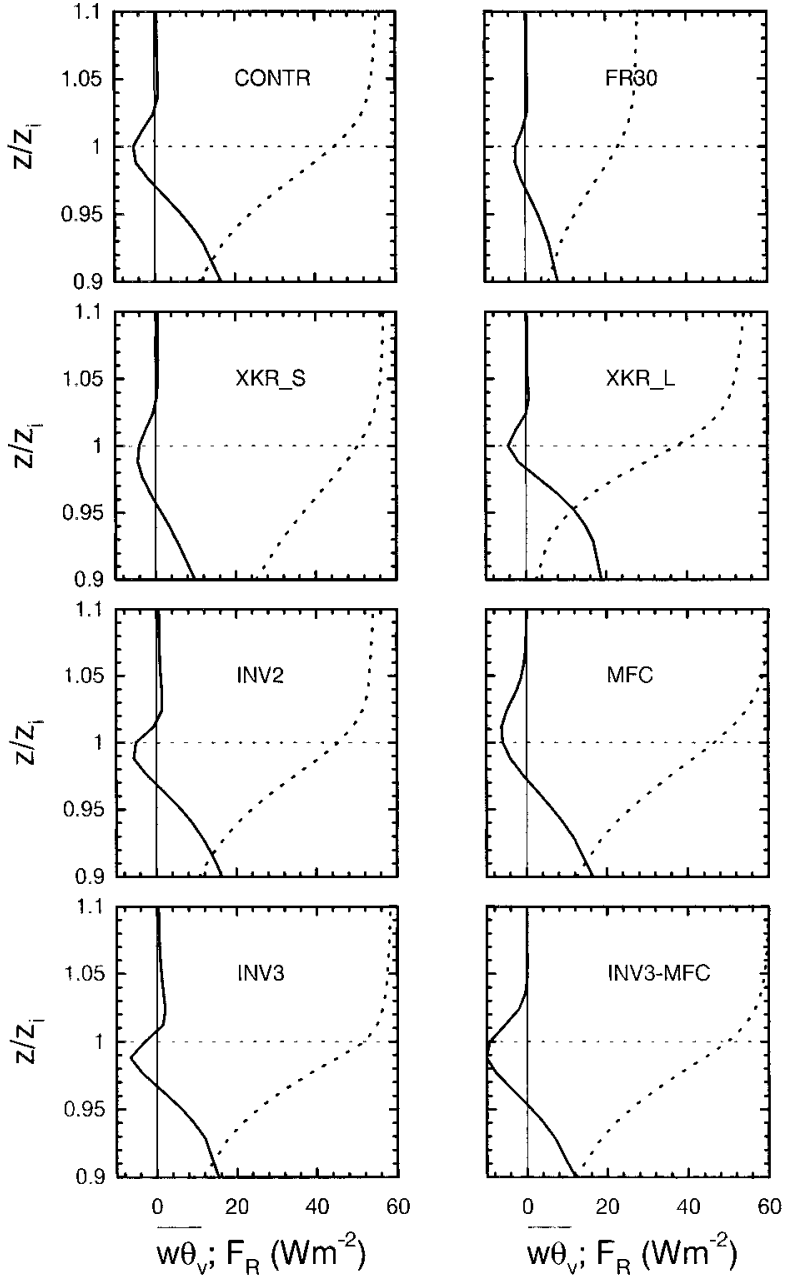

FIG. 8. Same as Fig. 7 but for the buoyancy flux (solid) and longwave radiative flux (dotted).

for this case because of the following reason. If we use $A^{\prime}=1$ for the INV3-MFC case, $A \sim 0.33$, which yields a $50 \%$ increase on the $A / \mathrm{Ri}_{c}$ term in (3.4) compared to using $A=0.2$. Because the $A / \mathrm{Ri}_{c}$ term contributes only about half of the total right-hand side of (3.4), as shown in the fourth and fifth columns of Table 3, a larger $A^{\prime}$ (from 0.5 to 1 ) for the INV3-MFC case increases the right-hand side of (3.4) by only about $25 \%$. In other words, with the contribution from the $\Delta F_{R}$ term, the entrainment rate prediction is not as sensitive to the $A^{\prime}$ value we set in the closure assumption.

If neglecting the $\Delta F_{R}$ term and plotting $w_{e} / w_{*_{c}}$ versus $A / \mathrm{Ri}_{c}$, we obtain Fig. 12a. All data points lie around the $A=0.5$ curve fit; this suggests that the simple parameterization $w_{e} / w_{* c}=A / \mathrm{Ri}_{c}$ also fits our LES solutions reasonably well but with an $A$ value that is about 2.5 times larger. This larger $A$ value is needed to compensate for the omission of the $\Delta F_{R}$ effect.

If we calculate $R_{i c}$ using the full temperature jump, that is, the temperature difference between the mixedlayer value and that above the turbulent layer, as used by previous studies (e.g., BR99), then all data points lie about the $w_{e} / w_{*_{c}} \sim 0.8 / \mathrm{Ri}_{c}$ curves as indicated in Fig. $12 \mathrm{~b}$. (The full temperature jump is about $1.5 \mathrm{~K}$ for all weaker inversion runs, about $2.8 \mathrm{~K}$ for INV2, and about $7.3 \mathrm{~K}$ for INV3 and INV3-MFC runs during the last 80 min of the simulation.) Our result is consistent with BR99 where they found $w_{e} / w_{* c} \sim 0.7 / \mathrm{Ri}_{c}$ for all highvertical-resolution smoke-cloud simulations.

There is about a $25 \%$ difference in the entrainment rate prediction between CONTR and MFC (also between INV3 and INV3-MFC) due to different numerics, implying a $25 \%$ uncertainty in our LESs for the entrainment rate prediction. LES with a monotone scheme tends to predict a larger entrainment rate, which is also found in BR99, and also gives a larger $\Delta F_{R}$ term. The latter is due to the fact that monotone scheme gives a more diffusive smoke concentration into the entrainment zone. Thus, both sides of (3.4) increase with the use of monotone schemes.

\section{4) Sensitivity tests}

To see if our results depend sensitively on the choice of the jump layer, we now choose $z_{\mathrm{fl}}$ and $z_{\mathrm{fl}}^{+}$levels differently. We define $z_{\mathrm{fl}}$ at the actual height where the minimum buoyancy flux occurs and $z_{\mathrm{fl}}^{+}$where the buoyancy flux becomes zero, as found from Fig. 8, so these heights are now different for different LES cases. For example, for INV3 we now use $z_{\mathrm{fl}}=0.99 z_{i}$ and $z_{\mathrm{fl}}^{+}=$ $1.01 z_{i}$ and for MFC $z_{\mathrm{fl}}=1.01 z_{i}$ and $z_{\mathrm{fl}}^{+}=1.07 z_{i}$. We then recompute the jumps, $\Delta \Theta$ and $\Delta F_{R}$, and all other parameters that depend on these jumps. This new set of

TABLE 2. LES output.

\begin{tabular}{lcccccc}
\hline \hline Experiment & $z_{i}(\mathrm{~m})$ & $w_{e}\left(\mathrm{~cm} \mathrm{~s}^{-1}\right)$ & $B\left(\mathrm{~W} \mathrm{~m}^{-2}\right)$ & $\overline{w \theta} \mid z_{\mathrm{fi}}\left(\mathrm{W} \mathrm{m}^{-2}\right)$ & $\Delta \Theta_{v}$ & $\Delta F_{R}\left(\mathrm{~W} \mathrm{~m}^{-2}\right)$ \\
\hline CONTR & 786.1 & 1.27 & 12.0 & -5.91 & $0.90(0.89)$ & $9.47(8.16)$ \\
FR30 & 747.6 & 0.73 & 5.3 & -2.92 & $0.94(1.00)$ & $4.40(5.64)$ \\
XKR-S & 767.8 & 0.98 & 11.6 & -5.73 & $1.02(1.06)$ & $5.87(7.30)$ \\
XKR-L & 804.8 & 1.58 & 10.1 & -4.74 & $1.05(0.92)$ & $15.05(11.72)$ \\
INV2 & 746.6 & 0.74 & 11.3 & -6.50 & $1.83(2.13)$ & $7.96(9.69)$ \\
INV3 & 717.0 & 0.35 & 11.4 & -7.41 & $4.22(2.88)$ & $6.00(7.98)$ \\
INV3-MFC & 734.9 & 0.45 & 9.7 & -10.73 & $4.15(5.27)$ & $10.50(14.36)$ \\
MFC & 788.8 & 1.58 & 12.2 & -6.86 & $1.05(0.99)$ & $12.58(8.81)$ \\
CONTR* & 776.6 & 1.10 & 14.8 & -6.15 & $1.02(1.05)$ & $4.90(8.35)$ \\
\hline
\end{tabular}


TABLE 3. LES output.

\begin{tabular}{|c|c|c|c|c|c|}
\hline Experiment & $w_{*_{c}}\left(\mathrm{~m} \mathrm{~s}^{-1}\right)$ & $\mathrm{Ri}$ & $w_{e} / w_{*_{c}}$ & $0.2 / \mathrm{Ri}$ & $\Delta F_{R} / \rho_{0} c_{p} w_{*_{c}} \Delta \Theta_{v}$ \\
\hline CONTR & 0.917 & $27.5(27.2)$ & 0.0139 & $0.0073(0.0074)$ & $0.0100(0.0088)$ \\
\hline FR30 & 0.687 & $48.7(51.8)$ & 0.0106 & $0.0041(0.0039)$ & $0.0060(0.0072)$ \\
\hline XKR-S & 0.899 & $31.6(32.9)$ & 0.0109 & $0.0063(0.0061)$ & $0.0056(0.0067)$ \\
\hline XKR-L & 0.872 & $36.3(31.8)$ & 0.0181 & $0.0055(0.0063)$ & $0.0144(0.0128)$ \\
\hline INV2 & 0.883 & $57.2(66.6)$ & 0.0084 & $0.0035(0.0030)$ & $0.0043(0.0045)$ \\
\hline INV3 & 0.874 & $129.4(88.3)$ & 0.0040 & $0.0015(0.0023)$ & $0.0014(0.0028)$ \\
\hline INV3-MFC & 0.835 & $142.9(181.5)$ & 0.0054 & $0.0014(0.0011)$ & $0.0027(0.0029)$ \\
\hline $\mathrm{MFC}$ & 0.923 & $31.8(30.0)$ & 0.0171 & $0.0063(0.0067)$ & $0.0114(0.0084)$ \\
\hline CONTR* & 0.979 & $27.0(27.8)$ & 0.0112 & $0.0074(0.0072)$ & $0.0043(0.0071)$ \\
\hline
\end{tabular}

values is given inside the parentheses in Tables 2 and 3. We then replot the right-hand side of (3.4) in Fig. 13. The results are similar to those of Fig. 11, showing that the $\Delta F_{R}$ term still contributes significantly to the entrainment rate.

The MFC and INV3 cases have the biggest changes in their $\Delta F_{R}$ term, mainly because of the difference in defining $z_{\mathrm{f}}$. The new jump layer of MFC is now from $1.01 z_{i}$ to about $1.07 z_{i}$. This slightly higher $z_{\mathrm{fl}}$ level results in a $\sim 30 \%$ reduction in $\Delta F_{R}$, and therefore a smaller right-hand side of (3.4). For INV3, the jump layer is now from $0.99 z_{i}$, which gives a larger $\Delta F_{R}$ term. The results are less sensitive to the location of $z_{\mathrm{fl}}^{+}$.

The new relationship between $0.2 / \mathrm{Ri}_{c}$ and $w_{e} / w_{* c}$ remains similar to that shown in Fig. 12a, again indicating the need to increase the $A$ value in order to compensate for the omission of the $\Delta F_{R}$ effect.

\section{d. Physical interpretation of $\Delta F_{R}$}

We have demonstrated that the radiative flux jump across the thin jump layer (from $z_{\mathrm{fl}}$ to $z_{\mathrm{fl}}^{+}$) is important

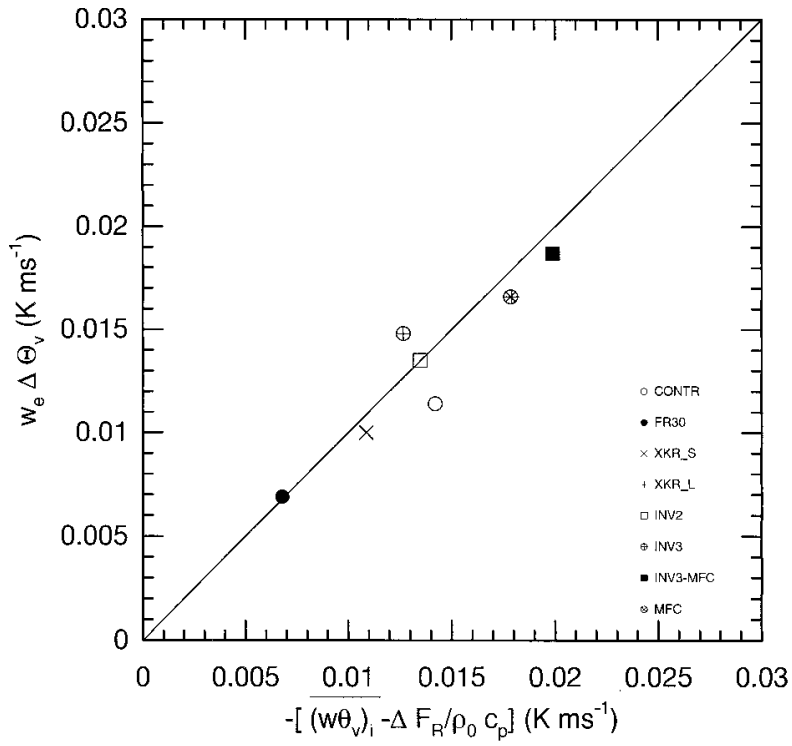

FIG. 9. The right- and left-hand sides of the jump condition, Eq. (3.2), calculated from the eight LESs. in the equation of the entrainment rate in a radiatively driven PBL, even though that part of radiative flux jump accounts for only $20 \%$ or less of the total jump, $F_{0}$. Since we do not include clear-air radiation in the simulations, all of the radiative flux divergence must exist inside the smoke region. The $\Delta F_{R}$ portion apparently results from smoke concentration between $z_{\mathrm{fl}}$ and $z_{\mathrm{fl}}^{+}$. Figure 14 shows the instantaneous smoke concentration in an $x-z$ cross section from the CONTR run. The shaded area has smoke concentrations larger than 0.5 , and the contour lines above the shaded region represent smoke concentration contours $s=0.4,0.3,0.2$, and 0.1 , respectively. Note that $z_{\mathrm{fl}}$ is close to the horizontally averaged (over the whole numerical domain) height of the $s=0.5$ contour line. So, about half of the local smokecloud tops, that is, $z_{\text {top }}(x, y)$, are above the mean $z_{\mathrm{fl}}$ level. We can see clearly there is a considerable amount of smoke above $z_{\mathrm{fl}}$, which leads to a nonzero $\Delta F_{R}$.

It is therefore clear that $\Delta F_{R}$, even though it shows up explicitly in the entrainment rate equation, represents radiative cooling inside the smoke (or cloud) region.

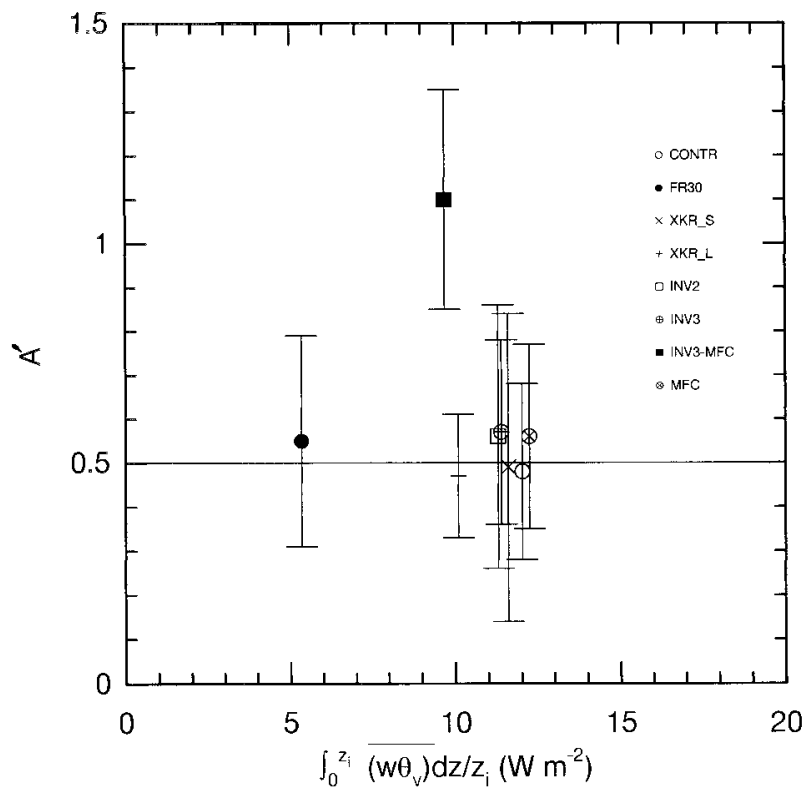

FIG. 10. The ratio $A^{\prime}$ vs the layer-averaged buoyancy flux from the eight LESs. 


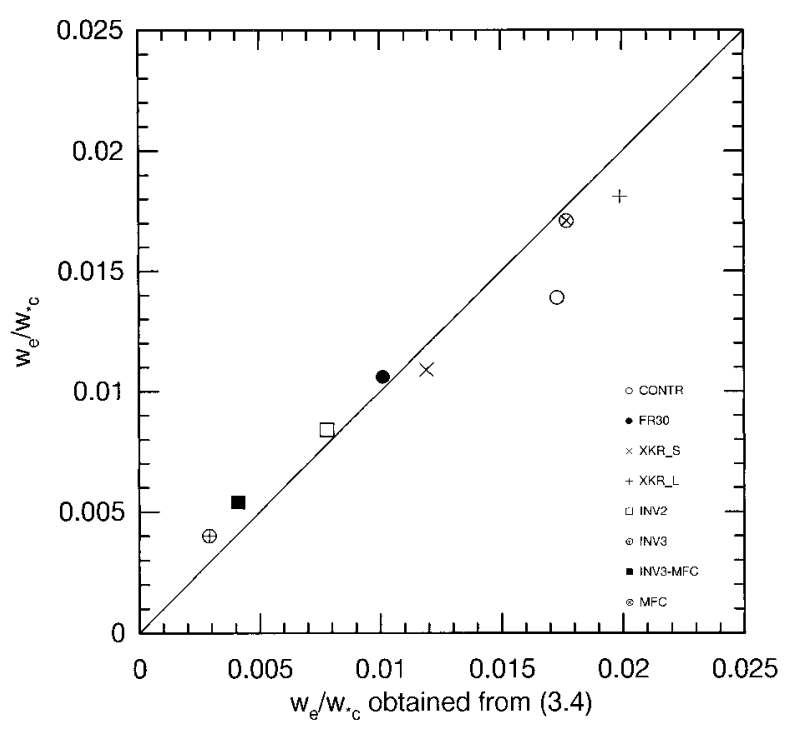

FIG. 11. The right-hand and left-hand sides of the entrainment equation, Eq. (3.4), calculated from the eight LESs.

The cooling does not exist in the clear air region above the smoke edge, so it does not promote entrainment by weakening the local inversion as has been previously argued. However, we should point out that in nature there does exist some longwave radiation in the clear air part of the inversion layer due to the temperature and water vapor distributions, but that clear-air radiative cooling is excluded in our simulations and should not be confused with the effect of $\Delta F_{R}$. Also, we believe that in nature the magnitude of the clear-air radiative flux divergence (or convergence) is much smaller than that of $\Delta F_{R}$. Since $\Delta F_{R}$ represents radiative cooling within cloud elements, we have no reason to think that it plays a different role in generating turbulence and in promoting entrainment compared to the other part of radiative cooling that exists below $z_{\mathrm{fl}}$, even though after averaging they show up in different equations.

For marine stratocumulus applications, we expect the $\Delta F_{R}$ term to play a smaller role in (3.4), because the "cloud" amount above $z_{\mathrm{fl}}$ is much smaller in stratocumulus cases than in smoke cloud cases. This is because cloud droplets evaporate quickly in the dry and warm environment in the inversion layer, leading to a nearly discontinuous liquid water mixing ratio across the local cloud top as often observed in stratocumulus. In the following section we will discuss applications of the entrainment rate equation to a smoke-cloud analog of stratocumulus, and with the understanding of the physical origin of the radiative flux divergence $\Delta F_{R}$ we will propose an analytical method to relate $\Delta F_{R}$ to the cloudtop fluctuations.

\section{Applications to marine stratocumulus topped PBLs}

Many studies of stratocumulus (e.g., Turton and Nicholls 1987; Bretherton and Wyant 1997) assumed that

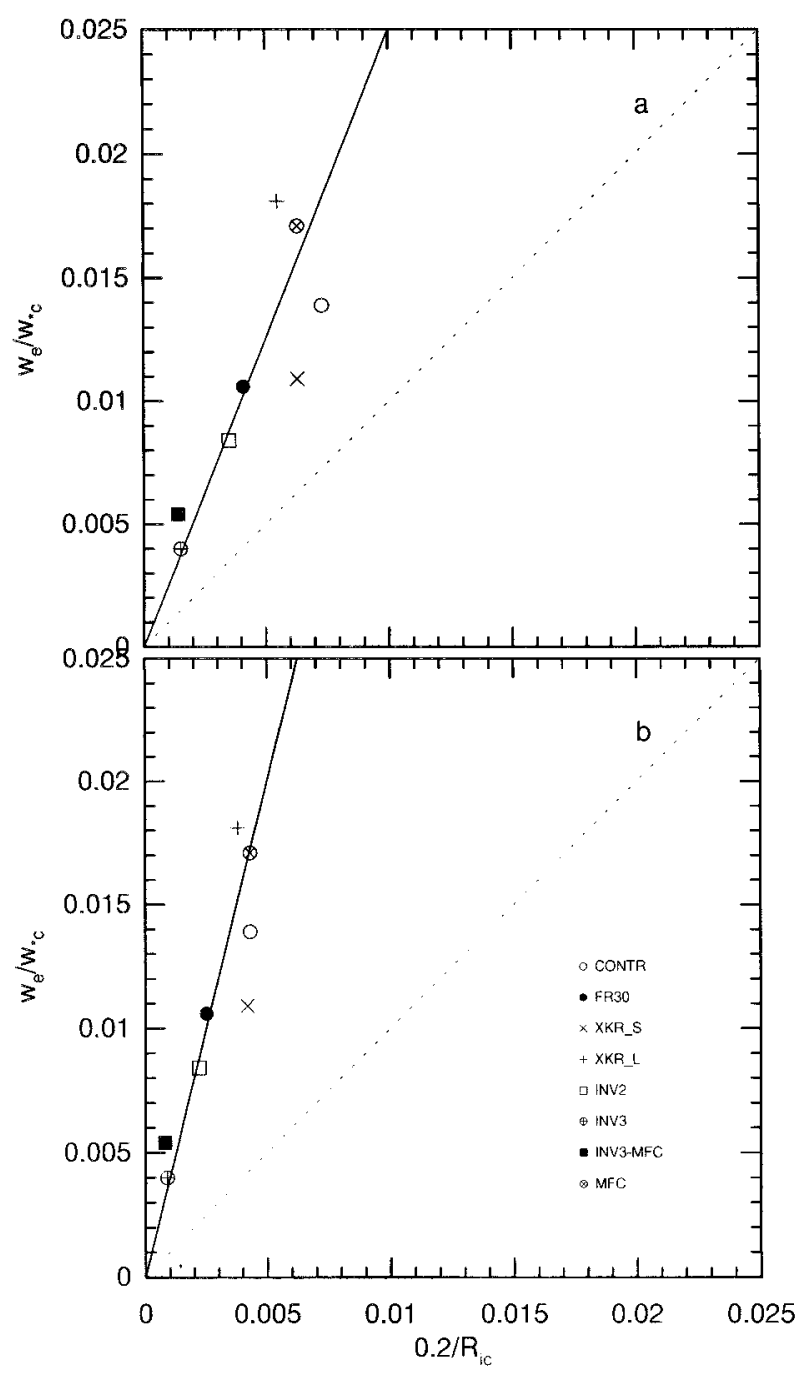

FIG. 12. The ratio $w_{e} / w_{* c}$ vs $0.2 / \mathrm{Ri}_{c}$ (a) where $\mathrm{Ri}_{c}$ is computed based on the buoyancy jump between $z_{\mathrm{fl}}$ and $z_{\mathrm{fl}}^{+}$and the solid line represents $w_{e} / w_{* c}=0.5 / \mathrm{Ri}_{c}$; and (b) where $\mathrm{Ri}_{c}$ is computed based on the full buoyancy jump and the solid line represents $w_{e} / w_{* c}=$ $0.8 / \mathrm{Ri}_{c}$.

the entrainment rate normalized by $w_{* c}$ depends only on the inverse of a bulk interfacial Richardson number, that is, $w_{e} / w_{* c}=A / \mathrm{Ri}_{c}$ (note their $\mathrm{Ri}_{c}$ is defined slightly differently from ours; their $\mathrm{Ri}_{c}$ is computed based on the full temperature jump). Most of the studies derived this simple entrainment rate equation based on simple scale analysis, which does not provide any physical insight into how and why radiation affects entrainment. In appendix $\mathrm{A}$, we derive an entrainment rate equation for stratocumulus using the same procedure as in section 3, which is similar to that by Deardorff (1976) and by Stage and Businger (1981). Through the derivation, we can see that three assumptions have to be made in order to arrive at an entrainment rate that can be expressed as $A / \mathrm{Ri}_{c}$. First, the entrainment buoyancy flux was assumed to be a fraction of the layer-averaged buoyancy 


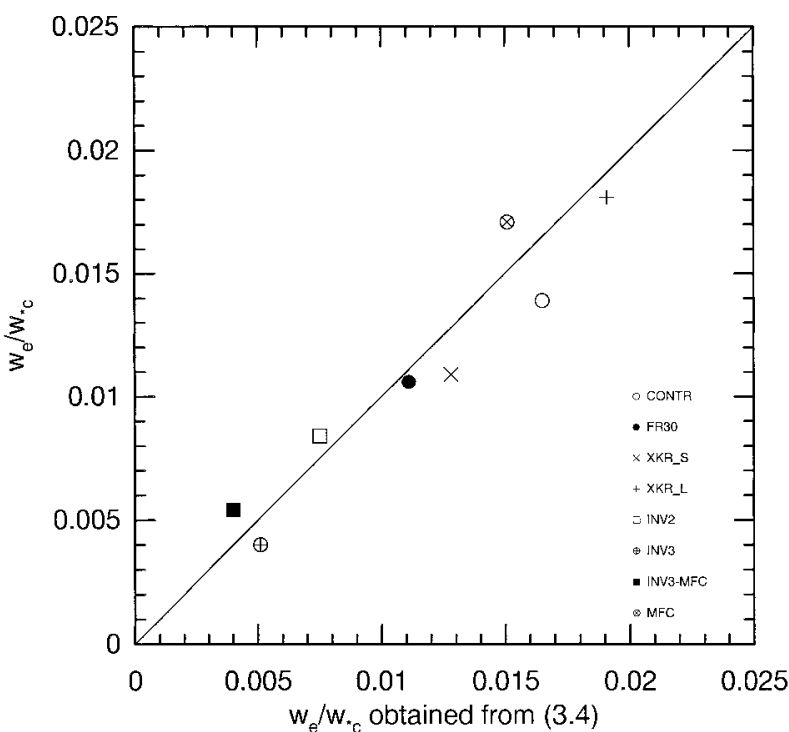

FIG. 13. Same as Fig. 11 but with different levels of $z_{\mathrm{fl}}$ and $z_{\mathrm{fl}}^{+}$.

flux. Second, all of the air parcels at $z_{\mathrm{fl}}$ were assumed to be saturated, which is clearly not the case because $z_{\mathrm{fl}}$ typically lies in the middle of cloud hummocks. Third, the $\Delta F_{R}$ term in (A.5) was neglected or assumed to be proportional to the inverse of $\mathrm{Ri}_{c}$. The first assumption was shown to work reasonably for the clear and smoke cloud cases, but how well it applies to stratocumulus should be investigated further. The second assumption looks illogical but how it affects the entrainment rate solution is not known. Here we will discuss only the third assumption.

To mimic a more realistic stratocumulus case, we generated another control case (CONTR*) in which we set a threshold value on the smoke-cloud concentration for it to become radiatively active. In this case, we use the threshold value $s=0.5$-the same value that defines the smoke-cloud top. In other words, we allow only smoke that is below $z_{\text {top }}(x, y)$ to be radiatively active.

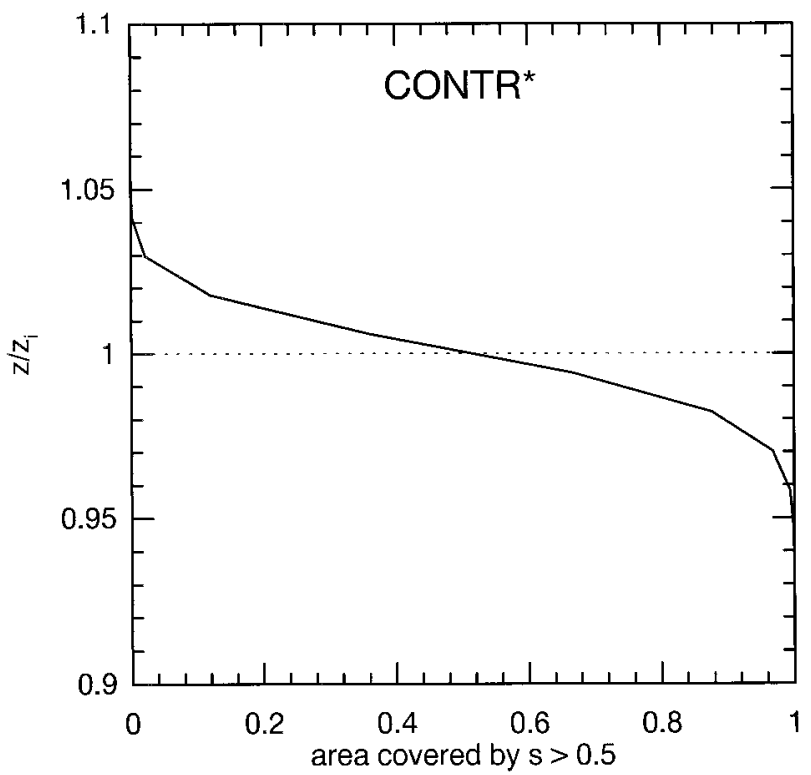

FIG. 15. The area coverage by smoke concentration larger than 0.5 in the entrainment zone from the CONTR* run.

Smoke that has a concentration less than 0.5 is excluded in the radiation calculation in (3.7). This is analogous to a real cloud case in that smoke with concentration larger than 0.5 can be seen as the cloudy region and that less than 0.5 as the cloud-free region. This results in a smaller $\Delta F_{R}$ because the local radiative flux becomes exactly zero above all local cloud tops. The only contribution to $\Delta F_{R}$ comes from the upper part of "cloud" hummocks.

It is not surprising that $z_{i}$ defined as the horizontal average of the $s=0.5$ isosurface agrees well with the $z_{\mathrm{fl}}$ level. As seen from Fig. 15 from CONTR*, the fractional area covered by this isosurface is about half at the $z_{i}$ level. And from the laboratory study by Deardorff et al. (1980), they showed that "the height of most

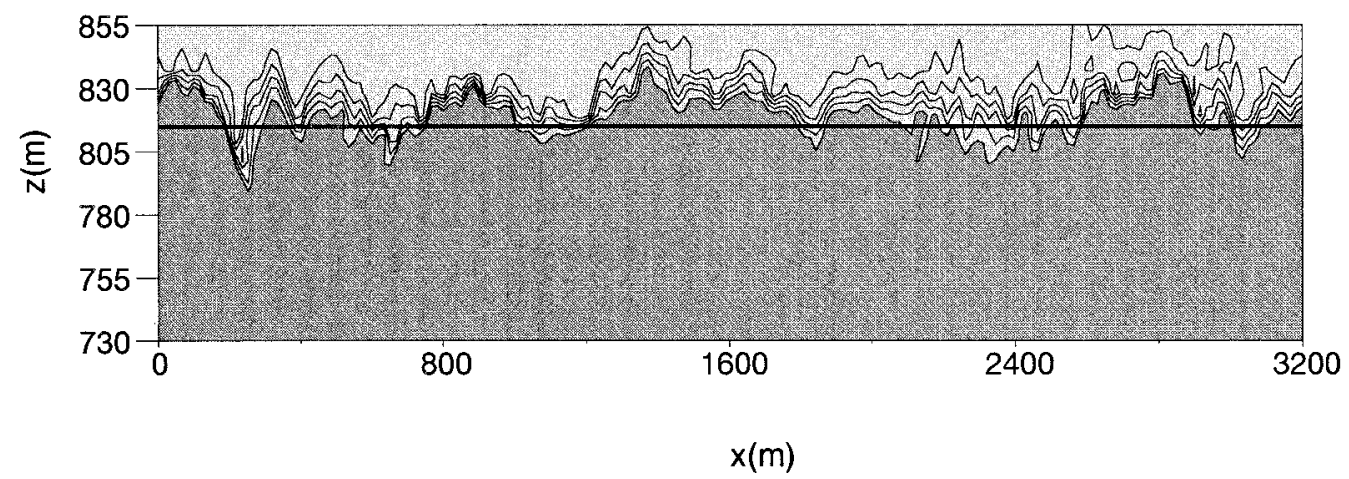

FIG. 14. A vertical cross section showing the smoke concentration field from the CONTR run. The shaded area includes smoke concentration larger than 0.5 and the contour lines above this area are for concentration levels 0.4 , $0.3,0.2$, and 0.1 , respectively. The $z_{\mathrm{fl}}$ level is indicated by the solid line while the $z_{\mathrm{fl}}^{+}$is close to the top of the plotted domain. 


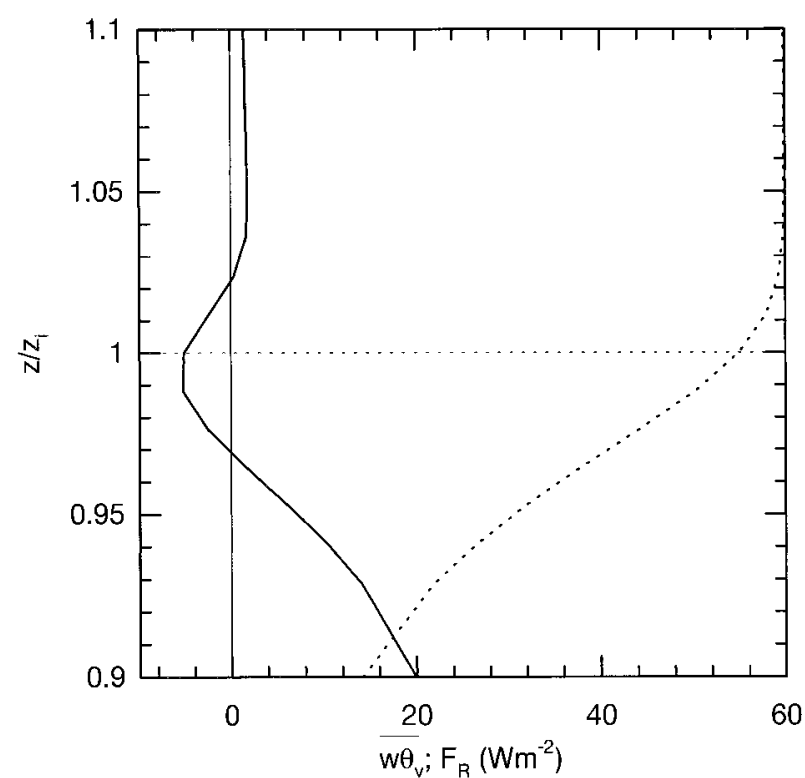

FIG. 16. Vertical distributions of the buoyancy flux (solid) and longwave radiative flux (dotted) in the entrainment zone from the CONTR* run. The straight dotted line indicates the bottom of the jump layer.

negative mean buoyancy flux is found to agree roughly with the height where mixed-layer fluid occupies onehalf the area." Thus, the $z_{\mathrm{fl}}$ level in stratocumulustopped PBLs is likely to occur at the level where cloudtop hummocks occupy about half of the area and the inversion air the other half.

Figure 16 shows the buoyancy and radiative fluxes of the CONTR* case, and the last row of Tables 1-3 reports those parameters. With only $\sim 5 \mathrm{~W} \mathrm{~m}^{-2}$ radiative jump in the jump layer in CONTR*, this radiative-fluxjump term still contributes significantly to the entrainment velocity. [Note that for a smoke cloud, which has no latent heating effects, $a=1$ and $b=0$ in (A.5).]

The cutoff of the smoke concentration level, used here in order to obtain a radiative cooling zone similar to that of a stratocumulus case, should vary depending on the amount of liquid water at the cloud top and the moisture in the inversion. As suggested by one of the reviewers, for a stratocumulus capped by a drier air, the cutoff should be larger than 0.5 and thus the contribution to the entrainment rate from $\Delta F_{R}$ could be even smaller.

Because $\Delta F_{R}$ exists within a thin layer at the cloud top, it may be difficult to measure in the field and may not be resolved in an LES that has a grid mesh too coarse to resolve cloud-top undulations. It is also impossible to obtain from any one-dimensional numerical model, which cannot simulate cloud-top undulations. For practical purposes, this radiative flux divergence needs to be related to quantities that are measurable. Giving the above physical interpretation of $\Delta F_{R}$, we were able to analytically relate this flux divergence to cloud-top fluctuations, as shown in appendix B. There

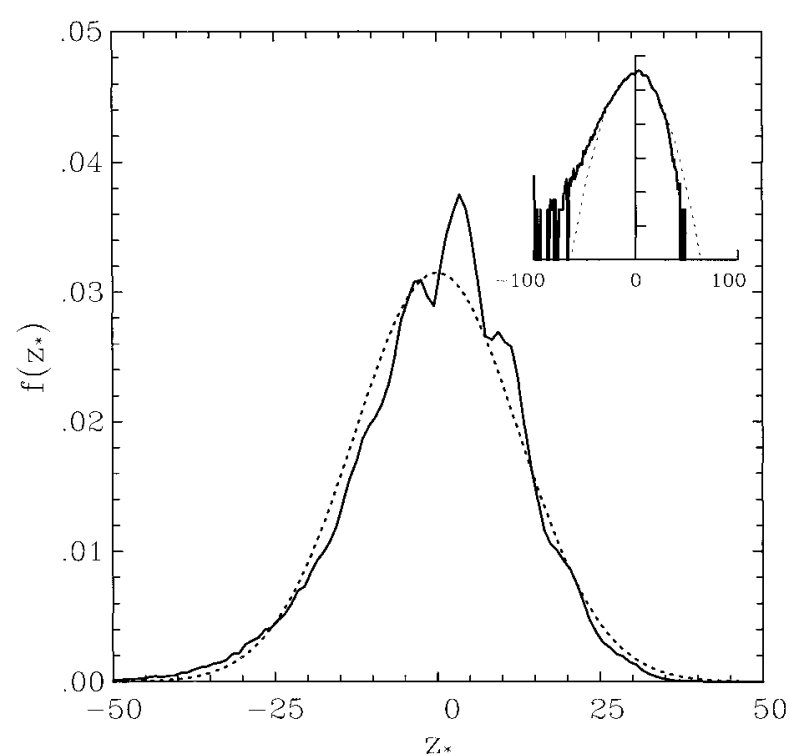

FIG. 17. The probability distribution of the smoke-top fluctuations from the CONTR run (solid) and of a Gaussian random variable (dotted). The inset shows the same figures but using a logarithmic scale in the abscissa.

we assumed that the cloud-top fluctuations are a Gaussian random variable (which is a reasonable assumption as evidenced from the LES solution, Fig. 17) and the radiative active trace species is piecewise constant $l o$ cally in any vertical column, that is, 1 below and 0 above the local cloud top. Then, the following analytical relationship can be derived to link $\Delta F_{R}$ to $\sigma_{z_{i}}$ (the standard deviation of cloud-top fluctuations) for a given $\kappa_{R}$ :

$$
\begin{aligned}
\Delta F_{R}=\frac{F_{0}}{2}\left\{1-e^{y^{2}}[1\right. & \\
& -\operatorname{erf}(y)]\}, \text { where } y=\frac{\rho_{0} \kappa_{R} \sigma_{z_{i}}}{\sqrt{2}} .
\end{aligned}
$$

For small $y$ (which one would expect for most marine stratocumulus cases), $\Delta F_{R}$ can be approximated by a linear function as shown in Fig. 18; that is,

$$
\Delta F_{R} / F_{0} \sim 0.46 y \text { for } y<0.3 .
$$

The standard deviation of cloud-top fluctuations $\sigma_{z_{i}}$ can be estimated from the LESs. Standard deviations are plotted as a function of the Richardson number from a variety of LESs in Fig. 19 (those clear-air PBL simulations denoted by crosses are from SU98); it shows a curve fit as

$$
\sigma_{z_{i}} / z_{i} \sim 0.6 R_{i c}^{-1}
$$

over a large range of PBL parameter space. One problem with this curve fit is that it gives an infinite large standard deviation of cloud-top fluctuations when $\mathrm{Ri}_{c}$ approaches zero. A more useful curve fit may take the 


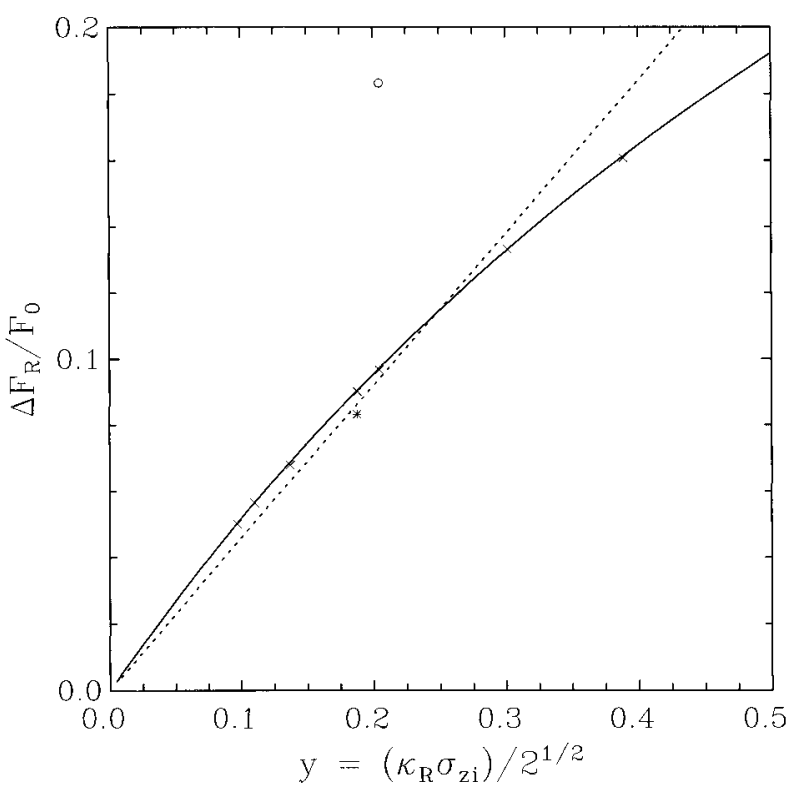

FIG. 18. The solid line shows the theoretically predicted longwave radiative flux jump from Eq. (B.8) as a function of $y$; the dashed line represents a curve fit of $\Delta F_{R} / F_{0}=0.46 y$; the $\times$ 's are calculated from Eq. (B.8) with $\sigma_{z_{i}}$ obtained from $z_{\text {top }}(x, y)$ of the LES runs; the open circle shows the flux jump directly from the CONTR run; and the asterisk shows the flux jump directly from the CONTR* run.

form $1 /\left(a+b \mathrm{Ri}_{c}\right)$ (D. A. Randall 1998, personal communication), but this requires knowing the asymptotic value of $\sigma_{z_{i}} / z_{i}$ when $\mathrm{Ri}_{c} \rightarrow 0$. For now, we will use (4.3) just to demonstrate the importance of the $\Delta F_{R}$ term in the entrainment rate equation. Using (4.2) and (4.3), (A.5) becomes

$$
\begin{aligned}
& \frac{w_{e}}{w_{* c}}=\frac{1}{R_{i c}} {\left[A+B \frac{a F_{0} z_{i}}{w_{* c}\left(a \Delta \Theta_{l}+b \Delta Q_{T}\right)}\right], } \\
& \text { where } \quad B=\frac{0.6\left(0.46 \kappa_{R}\right)}{c_{p} \sqrt{2}} \approx \frac{0.004}{c_{p}} .
\end{aligned}
$$

The importance of the radiation term increases as $\kappa_{R}$ increases (i.e., thinner radiative cooling zone), $a \Delta \Theta_{l}+$ $b \Delta Q_{T}$ decreases (weaker capping inversion), and $F_{0}$ increases (larger radiative forcing), consistent with that shown in section $3 c(2)$.

For most of our smoke-cloud simulations, $F_{0}=60$ $\mathrm{W} \mathrm{m} \mathrm{m}^{-2}, a=1, \Delta \Theta_{v} \sim 1 \mathrm{~K}, z_{i} \sim 750 \mathrm{~m}$, and $w_{* c} \sim 1$ $\mathrm{m} \mathrm{s}^{-1}$. Using these values in (4.4), we obtain $\sim 0.2$ for the second term in the bracket, which is close to the magnitude of the $A$ term. In other words, the radiative flux term has about the same magnitude as the inverse of the Richardson number term, consistent with what we showed in Table 3.

\section{Summary and discussion}

An entrainment rate equation can be derived based on (a) the cloud-top jump conditions and (b) a closure

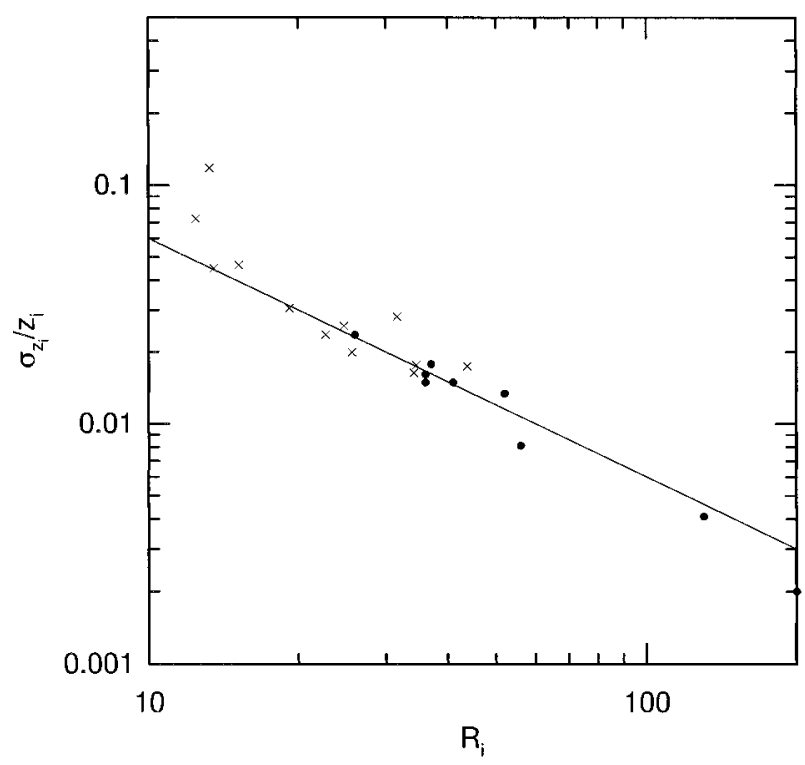

FIG. 19. Standard deviation of the cloud-top fluctuations, normalized by $z_{i}$, as a function of the interfacial Richardson number from the nine smoke-cloud runs (solid circles) and from several clear convective PBL runs reported in SU98 (crosses). The solid line shows a curve fit given in Eq. (B.9).

assumption that relates the entrainment buoyancy flux to the averaged buoyancy flux over the whole PBL. This led to an entrainment rate that depends not only on the inverse of interfacial Richardson number, as in the clear convective PBL, but also on the longwave radiative flux jump above the entrainment buoyancy flux level.

Smoke-cloud LESs were then used to check the relative importance of these two terms in determining the entrainment rate. From the eight smoke-cloud simulations, we found that the contribution from the radiative flux jump term is either larger than or about equal to the interfacial Richardson number term. Since smoke cannot evaporate like cloud droplets in the warm and dry inversion environment, the smoke-cloud LESs may exaggerate the importance of the radiative flux jump term. To see if the radiative flux jump term is still important for stratocumulus-like cases, we generated a smoke-cloud case that assumed all smoke concentration above a certain cutoff level to be radiatively inactive, in order to mimic the sharp drop of liquid water mixing ratio above stratocumulus. We found that the radiative flux term still contributes significantly to entrainment rate in this case.

The radiative flux divergence that appears explicitly in the entrainment rate equation actually exists within smoky (or cloudy) regions, just like any other part of the radiative flux divergence. It actually cools the cloudy air and thus enhances the local inversion strength; it does not make entrainment easier by reducing the inversion strength as has been previously argued. Mathematically it shows up in the entrainment equation, but physically it exists within the turbulent layer, cools the 
cloudy elements, and should therefore enhance turbulent mixing just like the other part of radiative flux divergence.

Using this physical understanding, and assuming a Gaussian distribution for the cloud-top fluctuations, we were able to analytically derive a relationship that links this radiative flux jump to the cloud-top fluctuations. The cloud-top fluctuations were then empirically related to the inverse of the interfacial Richardson number. Using this analytical expression for the radiative flux term, we showed analytically that both terms in the entrainment rate equation have about the same magnitude, which was consistent with our numerical results.

These results may explain partially why the ratio between $w_{e} / w_{*_{c}}$ and $\mathrm{Ri}_{c}^{-1}$ calculated from the GCSS smoke-cloud simulations reported in BR99 was at least 2 3 times larger than the ratio suggested for the clear convective PBL. We believe this larger ratio is needed in part to compensate for the omission of the contribution due to the $\Delta F_{R}$ term in (3.4).

Quantitatively, the simulated amount of $\Delta F_{R}$ is sensitive to numerics (centered finite differencing vs monotone schemes) or LES grid resolutions. A coarse grid LES that poorly resolves cloud-top undulations may generate spurious values of $\Delta F_{R}$. The question of how grid resolution and numerics affect the magnitude of $\Delta F_{R}$ is interesting, but is beyond the scope of this study. Here our main goal is to point out that $\Delta F_{R}$ exists physically within the cloudy region and yet can be a dominant term in the entrainment rate equation.

We realized, only after finishing the manuscript, that Deardorff (1981) gave the same argument. He stated that the portion of radiative flux divergence that exists in the entrainment zone, that is, $r$ in his notation, "should be defined in a manner consistent with the averaging method utilized in defining the turbulent fluxes," which in common practice is horizontal averaging. This averaging puts a portion of in-cloud radiative cooling inside the entrainment zone. He then argued that "a stratocumulus growth/decay model needs to treat the fraction of the overall radiative flux difference existing above the well-mixed height as a variable quantity."

We would also like to mention a similar study by LM99, which was submitted to Quart J. Roy. Meteor. Soc. for publication at about the same time as this manuscript. Through LES they also argued that the radiative flux divergence that appears explicitly in the entrainment rate equation exists inside the simulated smoke (or cloud) region, and that part of flux divergence is no different compared to the rest of the radiative flux divergence in physical sense in driving turbulence and entrainment.

The radiative flux jump term in the entrainment rate equation is likely to be smaller for stratocumulus, but whether it still plays an important role in directly determining entrainment rate and whether it can be parameterized as (4.2) requires LESs that can well resolve the cloud-top undulations.
Acknowledgments. We wish to thank Peter Duynkerke, Don Lenschow, Adrian Lock, Larry Mahrt, David Randall, Zbig Sorbjan, Margreet vanZanten, and the reviewers for their helpful discussions and suggestions. PPS's contributions are partially supported by ONR Government Order N00014-92-F-0117. NCAR is sponsored by the National Science Foundation.

\section{APPENDIX A}

\section{Entrainment Rate Equation for Stratocumulus-Topped PBL}

With latent heating the virtual potential temperature is no longer a conserved variable in a moist adiabatic process. We instead need to use a thermodynamic quantity that is conserved in a wet atmosphere, such as equivalent potential temperature, liquid potential temperature, or liquid water static energy, to derive a jump condition similar to (3.2). Here we choose liquid water moist static temperature $\theta_{l}$ defined as $\theta-\left(L / c_{p}\right) q_{l}$, where $\theta$ is the potential temperature, $q_{l}$ is the liquid water mixing ratio, $L$ the latent heat of condensation, and $c_{p}$ the specific heat of air at constant pressure. [Here $\theta_{l}$ is an approximate, linearized form of the liquid potential temperature, or is the liquid water static energy without the factor $c_{p}$. The following discussion remains the same no matter what conserved variable one chooses. The only difference is the thermal coefficients shown in (A.3).] Thus,

$$
\left.\overline{w \theta_{l}}\right|_{z_{\mathrm{fl}}}-\frac{1}{\rho_{0} c_{p}} \Delta F_{R}=-w_{e} \Delta \Theta_{l} .
$$

Similarly for the total mixing ratio $q_{T}$ :

$$
\left.\overline{w q_{T}}\right|_{z_{\mathrm{fl}}}=-w_{e} \Delta Q_{T},
$$

assuming no precipitation. Again, the capital-lettered $\Theta_{l}$ and $Q_{T}$ represent ensemble averages.

We apply the same closure assumption (3.3), that is, the entrainment buoyancy flux is assumed to be a fraction of the vertically averaged buoyancy flux, for the radiatively driven $\mathrm{PBL}$.

To use (3.3) in (A.1) and (A.2), we have to relate $\left.\overline{w \theta_{v}}\right|_{z_{\mathrm{fl}}}$ to $\left.\overline{w \theta_{l}}\right|_{z_{\mathrm{fl}}}$ and $\left.\overline{w q_{T}}\right|_{z \mathrm{fl}}$. Most often we assume that all air parcels at $z_{\mathrm{fl}}$ are saturated, which is of course not true because cloudy and clear air coexist at this cloudtop excursion level. Because representing the averaged buoyancy flux in a partly cloudy area is difficult (Randall 1987), most studies assume all air parcels at the $z_{\mathrm{fl}}$ level are saturated, which leads to the following relation between the entrainment buoyancy flux and the entrainment $\theta_{l}$ and $q_{T}$ fluxes:

$$
\left.\overline{w \theta_{v}}\right|_{z_{\mathrm{fl}}}=\left.a \overline{w \theta_{l}}\right|_{z_{\mathrm{fl}}}+\left.b \overline{w q_{T}}\right|_{z_{\mathrm{fl}}},
$$

where coefficients $a \equiv(1+1.609 \epsilon \gamma) /(1+\gamma)$ and $b \equiv$ $\left(L / c_{p}\right)(1-\epsilon+0.609 \epsilon \gamma) /(1+\gamma), \epsilon=c_{p} T_{0} / L, \gamma=$ $\left(L / c_{p}\right)\left(\partial q_{s} / \partial T\right), q_{s}$ is the saturation mixing ratio, and $T_{0}$ is a reference temperature. Here we use the approximate, 
linear relationships: $\theta_{v}=\theta+0.61 T_{0} q_{v}-T_{0} q_{l}$ and $q_{v}^{\prime} \sim\left(c_{p} \gamma / L\right) \theta^{\prime}$.

Using (3.3), (A.1), (A.2), and (A.3), we obtain

$$
\begin{gathered}
-w_{e}\left(a \Delta \Theta_{l}+b \Delta Q_{T}\right)+\frac{a}{\rho_{0} c_{p}} \Delta F_{R} \\
=-\frac{2 A}{1-A} \int_{0}^{z_{\mathrm{fl}}} \overline{w \theta_{v}} d z .
\end{gathered}
$$

Using the definition of $w_{* c}(3.5)$, we obtain

$$
\frac{w_{e}}{w_{* c}}=\frac{A}{\operatorname{Ri}_{c}}+\frac{a \Delta F_{R}}{\rho_{0} c_{p} w_{* c}\left[a \Delta \Theta_{l}+b \Delta Q_{T}\right]} .
$$

Here the interfacial Richardson number $\mathrm{Ri}_{c}$ is now

$$
\mathrm{Ri}_{c} \equiv\left(g / T_{0}\right) z_{\mathrm{fl}}\left(a \Delta \Theta_{l}+b \Delta Q_{T}\right) / w_{* c}^{2} .
$$

\section{APPENDIX B}

\section{Relating the Radiative Flux Jump to Cloud-Top Fluctuations}

Let us denote by $z_{*}$ the deviation of the local cloudtop height, $z_{\text {top }}$, from the mean cloud-top height $z_{i}$; that is,

$$
z_{*}=z_{\text {top }}-z_{i}, \text { where } z_{i}=\left\langle z_{\text {top }}\right\rangle .
$$

If $z_{*}$ is a centered Gaussian random variable with standard deviation $\sigma_{z_{i}}$, its density function $f\left(z_{*} ; \sigma_{z_{i}}\right)$ is

$$
\begin{array}{r}
f\left(z_{*} ; \sigma_{z_{i}}\right)=\frac{1}{\sigma_{z_{i}} \sqrt{2 \pi}} \exp \frac{-z_{*}^{2}}{2 \sigma_{z_{i}}^{2}}, \\
-\infty \leq z_{*} \leq \infty .
\end{array}
$$

In practice one may treat each vertical column at each time as an independent event, so $z_{*}$ may be thought of as a function of $(x, y)$ and integrations over $z_{*}$ may be thought of as integrations over $(x, y)$. However, in the subsequent discussion, and in keeping with the theory of probability, we will not make further reference to this event space.

If we assume that the smoke concentration $s\left(z, z_{*}\right)$ in any vertical column is a Heaviside function centered at $z_{\text {top }}=z_{*}+z_{i}$, then the local smoke concentration is unity for $z<z_{*}+z_{i}$ and zero elsewhere. Consequently the smoke path above some level $z, \mathcal{S}\left(z, z_{*}\right)$ is simply

$$
\mathcal{S}\left(z, z_{*}\right)= \begin{cases}z_{*}+z_{i}-z & \text { for } z<z_{*}+z_{i} \\ 0 & \text { otherwise, }\end{cases}
$$

whose expected value is

$$
\begin{aligned}
\left\langle\mathcal{S}\left(z ; \sigma_{z_{i}}\right)\right\rangle & =\int_{-\infty}^{\infty} \mathcal{S}\left(z, z_{*}\right) f\left(z_{*} ; \sigma_{z_{i}}\right) d z_{*} \\
& =\int_{z-z_{i}}^{\infty}\left(z_{*}+z_{i}-z\right) f\left(z_{*} ; \sigma_{z_{i}}\right) d z_{*} .
\end{aligned}
$$

At $z=z_{i}$ the expected value of the smoke path depends only on the breadth of the distribution function describing the cloud-top height as

$$
\left\langle\mathcal{S}\left(z_{i} ; \sigma_{z_{i}}\right)\right\rangle=\int_{0}^{\infty} \frac{z_{*}}{\sigma_{z_{i}} \sqrt{2 \pi}} \exp \left(\frac{-z_{*}^{2}}{2 \sigma_{z_{i}}^{2}}\right) d z_{*}=\frac{\sqrt{2} \sigma_{z_{i}}}{\pi} .
$$

Our entrainment formula requires some model for the jump in the radiative flux $\Delta F_{R}=F_{0}-\left\langle F\left(z_{i}\right)\right\rangle$. Thus we are interested in the expected value of the radiative flux at $z_{i}$. Equation (3.7) describes the dependence of the radiative flux $F(z ; c)$ on the value of the smoke path at $z$. Because, in our model, this smoke path depends on both $z$ and $z_{*}$, we will write the value of the radiative flux at some level $z$ as

$$
\begin{aligned}
& F\left(z, z_{*} ; \kappa_{R}\right) \\
& \quad= \begin{cases}F_{0} \exp \left[-\rho_{0} \kappa_{R} S\left(z, z_{*}\right)\right] & \text { for } z<z_{*}+z_{i} \\
F_{0} & \text { otherwise. }\end{cases}
\end{aligned}
$$

The expected value of $F$ is denoted by $\langle F(z)\rangle$ and is given by

$$
\langle F(z)\rangle=\int_{-\infty}^{\infty} F\left(z, z_{*} ; \kappa_{R}\right) f\left(z_{*} ; \sigma_{z_{i}}\right) d z_{*} .
$$

Using Eq. (B.3) in Eq. (B.6) and substituting above allows us (in the special case when $z_{*}$ is a centered Gaussian random variable) to solve for the expected value of the radiative flux analytically. At $z=z_{i}$ its expected value has a particularly simple form, which is only a function of the parameter $y=\rho_{0} \kappa_{R} \sigma_{z_{i}} / \sqrt{2}$ :

$$
\begin{aligned}
\left\langle F\left(z_{i} ; y\right)\right\rangle & =\frac{F_{0}}{2}+\frac{F_{0}}{\sigma_{z_{i}} \sqrt{2 \pi}} \int_{0}^{\infty} \exp \left(\frac{-z_{*}^{2}}{2 \sigma_{z_{i}}^{2}}-\kappa_{R} z_{*}\right) d z_{*} \\
& =\frac{F_{0}}{2}\left\{1+e^{y^{2}}[1-\operatorname{erf}(y)]\right\} .
\end{aligned}
$$

Here $\left\langle F\left(z_{i}\right)\right\rangle$ is a strictly decreasing function of $y$, which decreases from a maximum of $F_{0}$ at $y=0$ to $0.5 F_{0}$ as $y$ goes to infinity. In Fig. 18 we plot the fractional jump, $\Delta F_{R} / F_{0}$, predicted by Eq. (B.8) using the solid line. It has the expected behavior, decreasing as the optical thickness or thickness of the inversion zone decreases, increasing otherwise. For most of the experiments we use $\kappa_{R}=0.02$ and $\sigma_{z_{i}}$ is typically about $10 \mathrm{~m}$. Hence $y$ is generally less than 0.4 , and $\Delta F_{R} / F_{0}$ is well approximated by $0.46 y$ (i.e., the dashed line in Fig. 18).

Our ability to represent $\left\langle F\left(z_{i}\right)\right\rangle$ by an analytic form rests on three key assumptions. First, we assumed that cloud-top heights are well described by a Gaussian random variable; second, we assumed that the smoke concentrations were piecewise constant; and third, we took advantage of the simple analytic form for the radiative flux as a function of smoke path [e.g., Eq. (3.7)]. The third assumption is commonly made and is known to 
well represent the first-order effects of clouds on the longwave radiative fluxes. The second two assumptions are discussed further below.

In the simulations the cloud-top height tends to deviate from a pure Gaussian distribution, as values of the skewness in $z_{*}$ tend to be around -0.3 and the flatness is typically closer to 4 (for a Gaussian distribution these should be 0 and 3 , respectively). These departures from a pure Gaussian process can be seen in Fig. 17 which compares the actual distribution of cloud-top heights from experiment CONTR, with that predicted by a Gaussian distribution with the same variance. The deviation from a Gaussian distribution is mostly evident in the tails of the distribution and does not significantly impact our results. To substantiate this claim, we use the $z_{\text {top }}(x, y)$ data obtained by the LES and then artificially assume (as is done in the theory) that the smoke concentration is unity above and zero below $z=z_{\text {top }}$. We then solve for $\Delta F_{R}$ by numerically integrating $F_{R}$ over this distribution for each of the LES experiments. The results are shown by the X's in Fig. 18. The deviation of the $X$ 's from the solid line reflect the extent to which the deviation of the cloud-top heights from a Gaussian distribution impact the estimate of $\Delta F_{R}$. Clearly, from the perspective of the radiative calculations, the assumption that $z_{*}$ can be modeled as a Gaussian random variable is a good one.

The theory departs more substantially from the LES on the assumption that the smoke concentration is well described by a Heaviside function. The open circle in Fig. 18 shows that the model-derived value of $\Delta F_{R}$ for the control experiment departs significantly from the theory. Most of the disagreement is, however, due to the peculiar nature of the smoke cloud; smoke does not evaporate like water cloud droplets. In real clouds or in simulations of real clouds, the gradient in the radiatively active component (liquid water) is strongly constrained thermodynamically. Small amounts of water diffusing into the inversion typically evaporate quickly and hence do not become radiatively active until saturation is achieved. In other words, the liquid water content in real clouds is expected to vary much more sharply across cloud top. We mimic this cloud-top discontinuity in the smoke cloud by letting only smoke concentrations greater than 0.5 be radiatively active. This was done in experiment CONTR* and (as evidenced by the asterisk in Fig. 18) it leads to much better correspondence between the numerical simulation and our simple analytic model.

To close this theory, all that is needed is some way of estimating $\sigma_{z_{i}}$. On dimensional grounds one might expect $\sigma_{z_{i}}$ to scale inversely with the interfacial Richardson number of the flow. This expectation is supported by results from the LES. In Fig. 19 the value of $\sigma_{z_{i}} / z_{i}$ is plotted versus the Richardson number for experiments involving both smoke clouds and clear convective boundary layers (the latter cases were described in SU99). The experiments suggest that $\sigma_{z_{i}}$ is a decreasing function of Ri. If we want to relate the cloud-top fluctuations to the inverse of the Richardson number, the following curve fit (shown as the solid line in Fig. 19) is reasonable:

$$
\sigma_{z_{i}} / z_{i} \sim 0.6 R_{i}^{-1}
$$

\section{REFERENCES}

Beets, C., and B. Koren, 1996: Large-eddy simulation with accurate implicit subgrid-scale diffusion. Rep. NM-R9601, Department of Numerical Mathematics, Utrecht, the Netherlands, 24 pp.

Bretherton, C. S., and M. C. Wyant, 1997: Moisture transport, lower tropospheric stability, and decoupling of cloud-topped boundary layers. J. Atmos. Sci., 54, 148-167.

__ and Coauthors, 1999: An intercomparison of radiatively-driven entrainment and turbulence in a smoke cloud, as simulated by different numerical models. Quart. J. Roy. Meteor. Soc., in press.

Carson, D. J., 1973: The development of a dry inversion-capped convectively unstable boundary layer. Quart. J. Roy. Meteor. Soc., 99, 450-467.

Deardorff, J. W., 1974: Three-dimensional numerical study of turbulence in an entraining mixed layer. Bound.-Layer Meteor., 7, 199-226.

_ 1976: On the entrainment rate of a stratocumulus-topped mixed layer. Quart. J. Roy. Meteor. Soc., 102, 563-582.

_ 1980: Cloud-top entrainment instability. J. Atmos. Sci., 37, 131147.

_ 1981: On the distribution of mean radiative cooling at the top of a stratocumulus-capped mixed layer. Quart. J. Roy. Meteor. Soc., 107, 191-202.

- G. E. Willis, and B. H. Stockton, 1980: Laboratory studies of the entrainment zone of a convectively mixed layer. J. Fluid Mech., 100, 41-64.

Kahn, P. H., and J. A. Businger, 1979: The effect of radiative flux divergence on entrainment of a saturated convective boundary layer. Quart. J. Roy. Meteor. Soc., 105, 303-306.

Koren, B., 1993: A robust upwind discretization method for advection, diffusion and source terms. Notes on Numerical Fluid Mechanics, C. B. Vreugdenhil and B. Koren, Eds., Vol. 45, Vieweg, $117-138$.

Kraus, H., and E. Schaller, 1978: A note on the closure in Lilly-type inversion models. Tellus, 30, 284-288.

Lilly, D. K., 1968: Models of cloud-topped mixed layers under a strong inversion. Quart. J. Roy. Meteor. Soc., 94, 292-309.

— a cloud-topped mixed layer. J. Atmos. Sci., 37, 482-487.

Lock, A. P., and M. K. MacVean, 1999: The parameterization of entrainment driven by surface heating and cloud-top cooling. Quart. J. Roy. Meteor. Soc., in press.

Moeng, C.-H., 1984: A large-eddy-simulation model for the study of planetary boundary-layer turbulence. J. Atmos. Sci., 41, 20522062.

_ 1987: Large-eddy simulation of a stratus-topped boundary layer. Part II: Implications for mixed-layer modeling. J. Atmos. Sci., 44, 1605-1614.

— , and P. P. Sullivan, 1994: A comparison of shear- and buoyancydriven planetary boundary layer flows. J. Atmos. Sci., 51, 9991022.

— S. Shen, and D. A. Randall, 1992: Physical processes within the nocturnal stratus-topped boundary layer. J. Atmos. Sci., 49, 2384-2401.

_ planetary boundary layer: Intercomparison among different numerical codes. Bull. Amer. Meteor. Soc., 77, 261-278.

Nieuwstadt, F. T. M., and J. A. Businger, 1984: Radiative cooling near the top of a cloudy mixed layer. Quart. J. Roy. Meteor. Soc., 110, 1073-1078. 
Randall, D. A., 1980a: Conditional instability of the first kind upsidedown. J. Atmos. Sci., 37, 125-130.

,- 1980 b: Entrainment into a stratocumulus layer with distributed radiative cooling. J. Atmos. Sci., 37, 148-159.

- 1984: Buoyant production and consumption of turbulence kinetic energy in cloud-topped mixed layers. J. Atmos. Sci., 41, 402-413.

, 1987: Turbulent fluxes of liquid water and buoyancy in partly cloudy layers. J. Atmos. Sci., 44, 850-858.

Schubert, W. H., 1976: Experiments with Lilly's cloud topped mixed layer model. J. Atmos. Sci., 33, 436-446.

- J. S. Wakefield, E. J. Steiner, and S. K. Cox, 1979: Marine stratocumulus convection. Part I: Governing equations and horizontally homogeneous solutions. J. Atmos. Sci., 36, 1286-1307.

Sorbjan, Z., 1996: Effects caused by varying the strength of the capping inversion based on a large eddy simulation model of the shear-free convective boundary layer. J. Atmos. Sci., 53, 2015-2024.
Stage, S. A., and J. A. Businger, 1981: A model for entrainment into a cloud-topped marine boundary layer. Part II: Discussion of model behavior and comparison with other models. J. Atmos. Sci., 38, 2230-2242.

Stull, R. B., 1976: The energetics of entrainment across a density interface. J. Atmos. Sci., 33, 1260-1267.

Sullivan, P. P., J. C. McWilliams, and C.-H. Moeng, 1996: A grid nesting method for large-eddy simulation of planetary boundarylayer flows. Bound.-Layer Meteor., 80, 167-202.

- C.-H. Moeng, B. Stevens, and D. H. Lenschow, 1998: Structure of the entrainment zone capping the convective atmospheric boundary layer. J. Atmos. Sci., 55, 3042-3064.

Turton, D. J., and S. Nicholls, 1987: A study of the diurnal variation of stratocumulus using a multiple mixed layer model. Quart. J. Roy. Meteor. Soc., 113, 969-1009.

vanZanten, M. C., P. G. Duynkerke, and J. W. M. Cuijpers, 1999: Entrainment parameterization in convective boundary layers derived from large eddy simulations. J. Atmos. Sci., 56, 813-828. 